\title{
Relatively hyperbolic groups: geometry and quasi-isometric invariance
}

\author{
Cornelia Druţu
}

\begin{abstract}
In this paper it is proved that relative hyperbolicity is a quasi-isometry invariant. As byproducts of the arguments, simplified definitions of relative hyperbolicity are provided. In particular we obtain a new definition very similar to the one of hyperbolicity, relying on the existence of a central left coset of a peripheral subgroup for every quasi-geodesic triangle.
\end{abstract}

Mathematics Subject Classification (2000). 20F65, 20F69, 57N10.

Keywords. Relative hyperbolicity, rigidity, quasi-isometry.

\section{Contents}

1 Introduction . . . . . . . . . . . . . . . . . . 504

1.1 Rigidity result . . . . . . . . . . . . . . . . . . . . . . . . . . . . . . . . . . . . . . . . . . .

1.2 Metric and algebraic relative hyperbolicity . . . . . . . . . . . . . . . . . . . . . . . 505

1.3 New definitions of relative hyperbolicity . . . . . . . . . . . . . . . . . . . . . . . . . . . . . . . . . . .

1.4 Organization of the paper . . . . . . . . . . . . . . . . 508

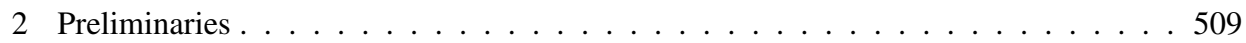

2.1 Definitions and notation . . . . . . . . . . . . . . . . . . . . . . . . . . . . . . . . . . . . . .

2.2 Asymptotic cones of a metric space . . . . . . . . . . . . . . . 510

3 Tree-graded metric spaces . . . . . . . . . . . . . . . . . . . . . . . . . . . . . . . . . . . . . .

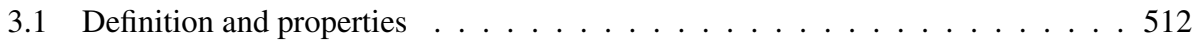

3.2 Topological bigons contained in pieces . . . . . . . . . . . . . . 513

4 Asymptotically tree-graded metric spaces . . . . . . . . . . . . . . . . . . . . . . . . . . . . . . . . . . . .

4.1 Definitions and properties . . . . . . . . . . . . . 516

4.2 Property $\left(T_{2}\right)$ and polygons with limit edges . . . . . . . . . . . . 520

4.3 New definitions, useful for the rigidity of relatively hyperbolic groups . . . . . 524

4.4 New definition, closer to the definition of hyperbolicity . . . . . . . . . . . 528

5 Quasi-isometric rigidity of relatively hyperbolic groups . . . . . . . . . . . . 538

References ............................. 544 


\section{Introduction}

1.1. Rigidity result. M. Gromov asked ([Gro87], [Gro93]) which properties of infinite finitely generated groups are invariant under quasi-isometry. Such properties are sometimes called geometric, while a class of groups defined by a geometric property is called rigid.

For instance, the class of virtually nilpotent groups is rigid [Gro81], while the class of virtually solvable groups is not rigid [Dyu00]; but smaller classes of virtually solvable groups are rigid ([FM98], [FM99], [EFW05]). Recall that a group is said to virtually satisfy a property $(\mathrm{P})$ if a finite index subgroup of it has property $(\mathrm{P})$.

Also, different classes of lattices of semisimple groups are rigid (this statement includes many deep results of different authors; see [Far97] and [Dru04] for surveys of these results).

The present paper focuses on the class of relatively hyperbolic groups. ${ }^{1}$ This notion was introduced by M. Gromov in [Gro87]. Other definitions, as well as developments of the theory of relatively hyperbolic groups can be found in [Bow97], [Far98], [Dah03b], [Yam04], [DS05b], [Osi06]. In $\S 1.2$ and $\S 1.3$ we discuss in more detail different ways to define relative hyperbolicity.

Beside hyperbolic groups, other examples of relatively hyperbolic groups are:

(1) fundamental groups of finite graphs of groups with finite edge groups; these groups are hyperbolic relative to the vertex groups [Bow97];

(2) fundamental groups of complete finite volume manifolds of pinched negative sectional curvature; these are hyperbolic relative to the fundamental groups of their cusps ([Bow97], [Far98]);

(3) fundamental groups of (non-geometric) Haken manifolds with at least one hyperbolic component; such groups are hyperbolic relative to fundamental groups of maximal graph-manifold components and to fundamental groups of tori and Klein bottles not contained in a graph-manifold component;

(4) fully residually free groups, also known as limit groups; it is proved in [Dah03a] that these groups are hyperbolic relative to their maximal Abelian non-cyclic subgroups; moreover they are $\mathrm{CAT}(0)$ with isolated flats [AB05].

There exist also interesting examples of groups displaying an "intermediate" version of relative hyperbolicity. Such groups are weakly relatively hyperbolic, not (strongly) relatively hyperbolic, but nevertheless they have some features in common with (strongly) relatively hyperbolic groups, for instance, their asymptotic cones have a tree-graded structure in the sense of [DS05b] (see Definition 3.1 in the present paper). Such groups are the mapping class groups of surfaces of complexity at least

\footnotetext{
${ }^{1}$ By relatively hyperbolic group we mean what is sometimes called in the literature strongly relatively hyperbolic group, in contrast with weakly relatively hyperbolic group.
} 
two ([Beh05], [BDM05]), fundamental groups of 3-dimensional graph manifolds ([KL98], [KKL98], [BDM05]), as well as many Artin groups ([KS04], [BDM05]).

Recently, relatively hyperbolic groups have been used to construct examples of infinite finitely generated groups with unusual properties. Thus in [Osi04] it is proved, using relatively hyperbolic groups, that there exist torsion-free two-generated groups with exactly two conjugacy classes.

Convention 1.1. Throughout the paper, relatively hyperbolic groups are assumed to be finitely generated and hyperbolic relative to finitely many proper finitely generated subgroups.

If a group $G$ is hyperbolic relative to some subgroups $H_{1}, \ldots H_{m}$ then the subgroups $H_{1}, \ldots, H_{m}$ are called peripheral subgroups.

The present paper answers affirmatively to the question whether relative hyperbolicity is a quasi-isometry invariant (Problem 1.15 in [DS05b]).

Theorem 1.2 (relative hyperbolicity is geometric, Theorem 5.7). Let $G$ be a group hyperbolic relative to a family of subgroups $H_{1}, \ldots, H_{n}$. If a group $G^{\prime}$ is quasiisometric to $G$ then $G^{\prime}$ is hyperbolic relative to $H_{1}^{\prime}, \ldots, H_{m}^{\prime}$, and each $H_{i}^{\prime}$ can be embedded quasi-isometrically in $H_{j}$ for some $j=j(i) \in\{1,2, \ldots, n\}$.

Rigidity has previously been proved for some sub-classes of relatively hyperbolic groups (with stronger versions of rigidity theorems): non-uniform lattices in rank one semisimple groups different from $\operatorname{SL}(2, \mathbb{R})$ [Sch96], fundamental groups of non-geometric Haken manifolds with at least one hyperbolic component ([KL95], [KL97]), fundamental groups of graphs of groups with finite edge groups [PW02].

Question 1.3. Can the conclusion of Theorem 1.2 be improved to: " $G$ ' is hyperbolic relative to $H_{1}^{\prime}, \ldots, H_{m}^{\prime}$, with each $H_{i}^{\prime}$ quasi-isometric to some $H_{j}, j=j(i)$ "?

This is known to hold only under extra hypotheses on $H_{j}$ ([DS05b], [BDM05]). The weakest such hypothesis is that every $H_{j}$ is not relatively hyperbolic in the sense of Convention 1.1 [BDM05].

The main steps in the proof of Theorem 1.2 are explained in what follows.

1.2. Metric and algebraic relative hyperbolicity. In order to study rigidity it is necessary to have a definition of relative hyperbolicity of a group only in terms of its Cayley graphs. Most definitions (except the ones in [DS05b] and in [Osi06]) use not only a Cayley graph of the group but also a metric space obtained from this graph by gluing to each left coset of a peripheral subgroup some geometric object (a hyperbolic horoball [Gro87], countably many edges with one common endpoint [Far98] etc.). 
We recall definitions from [DS05b]. A complete geodesic metric space $\mathbb{F}$ is treegraded with respect to a collection $\mathcal{P}$ of closed geodesic subsets (called pieces), if the following two properties are satisfied:

$\left(T_{1}\right)$ two different pieces have at most one point in common;

$\left(T_{2}\right)$ any simple non-trivial geodesic triangle is contained in one piece.

A metric space $X$ is asymptotically tree-graded with respect to a collection of subsets $\mathcal{A}$ if every asymptotic cone of $X$ is tree-graded with respect to the collection of limit sets of sequences in $\mathcal{A}$ (see Section 2.2 for definitions of asymptotic cones, and of limit sets). Equivalently, $X$ is asymptotically tree-graded with respect to $\mathcal{A}$ if the following three geometric properties are satisfied (for details see Theorem 4.1 in [DS05b], Theorem 4.9 in this paper):

$\left(\alpha_{1}\right)$ finite radius tubular neighborhoods of distinct elements in $\mathcal{A}$ are either disjoint or intersect in sets of uniformly bounded diameter;

$\left(\alpha_{2}\right)$ a geodesic with endpoints at distance at most one third of its length from a set $A$ in $\mathcal{A}$ intersects a tubular neighborhood of $A$ of uniformly bounded radius;

$\left(\alpha_{3}\right)$ any fat geodesic polygon is contained in a tubular neighborhood of a set $A$ in $\mathcal{A}$ of uniformly bounded radius (here the meaning of "fat" is the contrary of "thin" in its metric hyperbolic sense; see Definition 4.5).

The space $X$ is properly asymptotically tree-graded with respect to $\mathcal{A}$ if it is not contained in any finite radius tubular neighborhood of a subset in $\mathcal{A}$.

Convention 1.4. In what follows we assume that all asymptotically tree-graded metric spaces are properly asymptotically tree-graded.

The notion of asymptotically tree-graded metric space is a metric version for the relative hyperbolicity of groups. Other similar notions can be found in [BF01], and in [HK05] in the context of CAT(0) metric spaces. The fact that the metric definition is coherent with the definition for groups is illustrated by the following result.

Theorem 1.5 ([DS05b], Theorem 1.11 and Appendix). A finitely generated group $G$ is hyperbolic relative to $H_{1}, \ldots, H_{m}$ if and only if $G$ is asymptotically tree-graded with respect to the collection of left cosets $\mathscr{L}=\left\{g H_{i} \mid g \in G / H_{i}, i \in\{1,2, \ldots, m\}\right\}$.

The equivalence in Theorem 1.5 suggests the following question, which appears as Problem 1.16 in [DS05b]: if a group is asymptotically tree-graded in a metric sense, that is, with respect to a collection of subsets $\mathcal{A}$, does it follow that it is relatively hyperbolic with respect to some finite family of subgroups ? The implication was previously known to be true only under some restrictive metric conditions on $\mathcal{A}$ (see [DS05b, Theorem 5.13] and [BDM05]).

We answer this question in the affirmative. 
Theorem 1.6 (Theorem 5.4). Let $G$ be an infinite finitely generated group asymptotically tree-graded with respect to a collection of subsets $\mathcal{A}$. Then $G$ is relatively hyperbolic with respect to some subgroups $H_{1}, \ldots, H_{m}$, such that every $H_{i}$ is contained in a bounded radius tubular neighborhood of a set $A_{i} \in \mathcal{A}$.

Theorem 1.6 implies Theorem 1.2. Indeed, a group quasi-isometric to a relatively hyperbolic group is asymptotically tree-graded as a metric space with respect to the images by quasi-isometry of the left cosets of peripheral subgroups [DS05b, Theorem 5.1].

Theorem 1.6 is also used in [BDM05] to prove the following result: given a group $G$ hyperbolic relative to $H_{1}, \ldots, H_{n}$, every quasi-isometric embedding into $G$ of a group which is not relatively hyperbolic has its image in a tubular neighborhood of bounded radius of a left coset $g H_{i}$; moreover the radius of the neighborhood depends only on $G, H_{1}, \ldots, H_{n}$ and on the constants of quasi-isometry, not on the domain of the quasi-isometry [BDM05, Theorem 4.1].

From this it is deduced in [BDM05] that in Theorem 1.2 under the extra assumption that each peripheral subgroup $H_{i}$ is not relatively hyperbolic the rigidity result holds, with the stronger conclusion that each $H_{i}^{\prime}$ is quasi-isometric to some $H_{j}$. This generalizes previous results from [DS05b].

An outline of the proof of Theorem 1.6 will be given in the following sections.

Theorem 1.6 is optimal in the sense that if the group $G$ and the collection $\mathcal{A}$ satisfy less properties than those required of asymptotically tree-graded metric spaces then the group $G$ may not be relatively hyperbolic. This is illustrated by the examples of groups constructed in [BDM05, §7.1] and in [OOS06]. These groups are not relatively hyperbolic, although they do contain a collection of subsets $\mathcal{A}$ such that all the asymptotic cones of the group are tree-graded with respect to some limits of sequences in $\mathcal{A}$. In each cone, not all the limits of sequences in $\mathcal{A}$ are considered as pieces though: there are limits which are geodesic lines, and different such lines intersect in more than one point. The subsets in $\mathcal{A}$ do not satisfy property $\left(\alpha_{1}\right)$ requiring uniformly bounded diameter for intersections of bounded radius tubular neighborhoods of different subsets in $\mathcal{A}$.

1.3. New definitions of relative hyperbolicity. If a group has an asymptotically tree-graded structure equivariant with respect to left translations, then a standard argument shows that the group is relatively hyperbolic (Proposition 5.1). Thus, the main step in the proof of Theorem 1.6 is to construct an equivariant asymptotically treegraded structure on a group out of an arbitrary asymptotically tree-graded structure. A natural idea is to consider all the translated asymptotically tree-graded structures $g \mathcal{A}=\{g A \mid A \in \mathcal{A}\}$ of a given asymptotically tree-graded structure $\mathcal{A}$ on a group $G$, and to take non-empty intersections of the form $\bigcap_{g \in G} g A_{g}$, with $A_{g} \in \mathcal{A}$. To make such an argument work, it is necessary that the asymptotically tree-graded proper- 
ties behave well with respect to intersections. The modification of the list of three geometric properties defining an asymptotically tree-graded metric space given in Theorem 4.22 ensures this good behavior with respect to intersections.

Asymptotically tree-graded metric spaces have a property that strongly reminds of hyperbolic metric spaces. A metric space is hyperbolic if and only if the edges of every quasi-geodesic triangle intersect a ball of uniformly bounded radius [Gro87, $\S 6]$. A space $X$ that is asymptotically tree-graded with respect to a collection of subsets $\mathcal{A}$ satisfies a similar property, called property $(*)$ (see Definition 4.27$)$. If $(X, \mathcal{A})$ satisfy only property $(*)$ then the space $X$ is called $(*)$-asymptotically treegraded with respect to $\mathcal{A}$. This notion is weaker than the notion of asymptotically tree-graded metric space (see Remark 4.29, (2)).

Property $(*)$ was essential in the proof of the fact that the Rapid Decay property transfers from the peripheral subgroups $H_{1}, \ldots, H_{m}$ of a relatively hyperbolic group to the group itself [DS05a]. A version of property $(*)$ in the context of CAT $(0)$ spaces appears in [Hru04], where it is called the Relatively Thin Triangle Property.

A natural question to ask is under which additional conditions is a $(*)$-asymptotically tree-graded metric space also asymptotically tree-graded. The arguments used to prove Theorem 4.22 can be adapted to answer this question.

Theorem 1.7 (Theorem 4.30). Let (X, dist) be a geodesic metric space and let $\mathcal{A}$ be a collection of subsets of $X$. The metric space $X$ is asymptotically tree-graded with respect to $\mathcal{A}$ if and only if $(X, \mathcal{A})$ satisfy properties $\left(\alpha_{1}\right)$ and $\left(\alpha_{2}\right)$, and moreover $X$ is $(*)$-asymptotically tree-graded with respect to $\mathcal{A}$.

1.4. Organization of the paper. Section 2 contains preliminaries on asymptotic cones, as well as notation.

In Section 3 are recalled some basic facts about tree-graded spaces. Proposition 3.9, proved in this section, is very useful in arguments deducing the general property $\left(T_{2}\right)$ from $\left(T_{1}\right)$ combined with $\left(T_{2}\right)$ restricted to particular types of geodesic triangles (see for instance property $\left(\Pi_{3}\right)$ below).

Section 4 begins with a short overview of properties of asymptotically tree-graded metric spaces. In $\S 4.2$ an induction argument and Proposition 3.9 are used to show the following central result. Denote by $\left(\Pi_{3}\right)$ the property $\left(T_{2}\right)$ restricted to triangles with edges limits of sequences of geodesics. If in an asymptotic cone $\operatorname{Con}_{\omega}(X)$ of a metric space $X$ a collection $\mathcal{A}_{\omega}$ of closed subsets satisfies $\left(T_{1}\right)$ and $\left(\Pi_{3}\right)$ then $\mathcal{A}_{\omega}$ satisfies $\left(T_{2}\right)$ in full generality (Corollary 4.19).

This statement is the main ingredient in the proof of Theorem 4.22, given in $\S 4.3$. It also plays a central part in the proof of Theorem 1.7 given in $\S 4.4$. Another difficult step in the proof of Theorem 1.7 is to deduce from properties $(*),\left(\alpha_{1}\right)$ and $\left(\alpha_{2}\right)$ the fact that fat quadrilaterals are contained in finite radius tubular neighborhoods of subsets in $\mathcal{A}$ (Lemma 4.33). Once this last statement is proved, from it as well as 
from property $(*)$ and Proposition 3.9 can be deduced property $\left(\Pi_{3}\right)$. Corollary 4.19 allows to finish the argument.

Theorem 1.6 is proved in Section 5. The first and most difficult step of the proof is to construct from a given asymptotically tree-graded structure on a group an equivariant asymptotically tree-graded structure. The subsets in the new equivariant asymptotically tree-graded structure are indexed by equivalence classes of fat hexagons. A simple argument then shows that the existence of an equivariant asymptotically tree-graded structure implies that the group is relatively hyperbolic (Proposition 5.1). This completes the proof of Theorem 1.6 and thus of Theorem 1.2.

Acknowledgement. The author wishes to thank the referee for numerous useful comments. Thanks are also due to Mark Sapir and Jason Behrstock for remarks that helped improving the exposition in the paper.

\section{Preliminaries}

2.1. Definitions and notation. Let $Y$ be a subset in a metric space ( $X$, dist). We denote by $\mathcal{N}_{\delta}(Y)$ the set $\{x \in X \mid \operatorname{dist}(x, Y)<\delta\}$, which we call the $\delta$-tubular neighborhood of $Y$. We denote by $\overline{\mathcal{N}}_{\delta}(Y)$ the set $\{x \in X \mid \operatorname{dist}(x, Y) \leq \delta\}$, called the $\delta$-closed tubular neighborhood of $Y$.

When $Y$ is a singleton $y$, we also use the notation $B(y, \delta)$ and respectively $\bar{B}(y, \delta)$.

Definition 2.1. An action of a group $G$ on a metric space $X$ is called $\mathcal{K}$-transitive, where $\mathcal{K}$ is a non-negative constant, if for every $x \in X$ the closed tubular neighborhood $\overline{\mathcal{N}}_{\mathcal{K}}(G x)$ of the orbit of $x$ coincides with $X$.

An $(L, C)$-quasi-isometric embedding of a metric space $\left(X, \operatorname{dist}_{X}\right)$ into a metric space $\left(Y, \operatorname{dist}_{Y}\right)$ is a map $q: X \rightarrow Y$ such that for every $x_{1}, x_{2} \in X$,

$$
\frac{1}{L} \operatorname{dist}_{X}\left(x_{1}, x_{2}\right)-C \leq \operatorname{dist}_{Y}\left(\mathfrak{q}\left(x_{1}\right), \mathfrak{q}\left(x_{2}\right)\right) \leq L \operatorname{dist}_{X}\left(x_{1}, x_{2}\right)+C,
$$

for some constants $L \geq 1$ and $C \geq 0$.

If moreover $Y$ is contained in the $C$-tubular neighborhood of $\mathfrak{q}(X)$ then $\mathfrak{q}$ is called an $(L, C)$ - quasi-isometry. In this case there exists an $(L, C)$-quasi-isometry $\overline{\mathfrak{q}}: Y \rightarrow X$ such that $\overline{\mathfrak{q}} \circ \mathfrak{q}$ and $\mathfrak{q} \circ \overline{\mathfrak{q}}$ are at uniformly bounded distance from the respective identity maps [GdlH90]. The quasi-isometry $\bar{q}$ is called quasi-converse of $q$.

If $q:[a, b] \rightarrow X$ is an $(L, C)$-quasi-isometric embedding then $\mathfrak{q}$ is called an $(L, C)$-quasi-geodesic (segment) in $X$. The same name is used for the image of $q$.

Notation 2.2. For every quasi-geodesic segment $\mathfrak{q}$ in a metric space $X$, we denote the origin of $\mathfrak{q}$ by $\mathfrak{q}_{-}$and the endpoint of $\mathfrak{q}$ by $\mathfrak{q}_{+}$. 
If $\mathfrak{q}_{i}:\left[0, \ell_{i}\right] \rightarrow X, i=1,2$, are two quasi-geodesic segments with $\mathfrak{q}_{1}\left(\ell_{1}\right)=$ $\mathfrak{q}_{2}(0)$, then we denote by $\mathfrak{q}_{1} \sqcup \mathfrak{q}_{2}$ the map $\mathfrak{q}:\left[0, \ell_{1}+\ell_{2}\right] \rightarrow X$ defined by $\mathfrak{q}(t)=$ $\mathfrak{q}_{1}(t)$ for $t \in\left[0, \ell_{1}\right]$ and $\mathfrak{q}(t)=\mathfrak{q}_{2}\left(t-\ell_{1}\right)$ for $t \in\left[\ell_{1}, \ell_{1}+\ell_{2}\right]$.

If an $(L, C)$-quasi-geodesic $q$ is $L$-Lipschitz then $q$ is called an $(L, C)$-almost geodesic.

2.2. Asymptotic cones of a metric space. The notion of asymptotic cone of a metric space was used implicitly in [Gro81], and it was defined in full generality and studied in [dDW84] and [Gro93]. For the definition, one needs the notion of non-principal ultrafilter. This is a finitely additive measure $\omega$ defined on the set of all subsets of $\mathbb{N}$ (or, more generally, of a countable set) and taking values in $\{0,1\}$, such that $\omega(F)=0$ for every finite subset $F$ of $\mathbb{N}$.

Convention 2.3. Throughout the paper all ultrafilters are non-principal, therefore we will omit mentioning it each time.

Notation 2.4. Let $A_{n}$ and $B_{n}$ be two sequences of objects and let $\mathcal{R}$ be a relation that can be established between $A_{n}$ and $B_{n}$ for every $n \in \mathbb{N}$. We write $A_{n} \mathcal{R}_{\omega} B_{n}$ if and only if $A_{n} \mathcal{R} B_{n} \omega$-almost surely, that is,

$$
\omega\left(\left\{n \in \mathbb{N} \mid A_{n} \mathcal{R} B_{n}\right\}\right)=1 .
$$

Examples: $=_{\omega},<_{\omega}, \subset_{\omega}$.

Given an ultrafilter $\omega$, an $\omega$-limit $\lim _{\omega} x_{n}$ of a sequence $\left(x_{n}\right)$ in a topological space $X$ is an element $x \in X$ such that for every neighborhood $\mathcal{N}$ of $x, x_{n} \in_{\omega} \mathcal{N}$. In a Hausdorff separable space if the $\omega$-limit of a sequence exists then it is unique. If $\left(x_{n}\right)$ is contained in a compact space then it has an $\omega$-limit [Bou65].

Given a space $X$ one can define its ultrapower $X^{\omega}$ as the quotient $X^{\mathbb{N}} / \approx$, where $\left(x_{n}\right) \approx\left(y_{n}\right)$ if $x_{n}={ }_{\omega} y_{n}$.

Let now $\left(X\right.$, dist) be a metric space, $e$ an element in its ultrapower $X^{\omega},\left(e_{n}\right)$ a representative of $e$, and $d=\left(d_{n}\right)$ a sequence of numbers in $(0,+\infty)$ such that $\lim _{\omega} d_{n}=+\infty$. Consider

$$
\S_{e}(X)=\left\{\left(x_{n}\right) \in X^{\mathbb{N}} \mid \text { there exists } M_{x} \text { such that } \operatorname{dist}\left(x_{n}, e_{n}\right) \leq{ }_{\omega} M_{x} d_{n}\right\} .
$$

Define the equivalence relation

$$
\left(x_{n}\right) \sim\left(y_{n}\right) \Longleftrightarrow \lim _{\omega} \frac{\operatorname{dist}\left(x_{n}, y_{n}\right)}{d_{n}}=0 .
$$

The quotient space $\wp_{e}(X) / \sim$ is denoted by $\operatorname{Con}_{\omega}(X ; e, d)$ and it is called the asymptotic cone of $X$ with respect to the ultrafilter $\omega$, the scaling sequence $d$ and 
the sequence of observation centers $e$. It is endowed with the natural metric dist $\omega$ defined by

$$
\operatorname{dist}_{\omega}(x, y)=\lim _{\omega} \frac{\operatorname{dist}\left(x_{n}, y_{n}\right)}{d_{n}} .
$$

Every asymptotic cone is a complete metric space.

A sequence of subsets $\left(A_{n}\right)$ in $X$ gives rise to a limit subset in the cone, defined by

$$
\lim _{\omega}\left(A_{n}\right)=\left\{\lim _{\omega}\left(a_{n}\right) \mid a_{n} \in_{\omega} A_{n}\right\} .
$$

If $\lim _{\omega} \frac{\operatorname{dist}\left(e_{n}, A_{n}\right)}{d_{n}}=+\infty$ then $\lim _{\omega}\left(A_{n}\right)=\emptyset$. Every non-empty limit subset $\lim _{\omega}\left(A_{n}\right)$ is closed.

If each set $A_{n}$ is a geodesic $\mathrm{g}_{n}$ with length of order $O\left(d_{n}\right)$ and $\lim _{\omega}\left(\mathrm{g}_{n}\right)$ is nonempty, then it is a geodesic in $\operatorname{Con}_{\omega}(X ; e, d)$. Therefore if $X$ is a geodesic space then every asymptotic cone of it is geodesic.

Definition 2.5. We call a geodesic in $\operatorname{Con}_{\omega}(X ; e, d)$ which appears as $\lim _{\omega}\left(\mathfrak{g}_{n}\right)$ with $\mathfrak{g}_{n}$ geodesics in $X$ a limit geodesic.

Not every geodesic in $\operatorname{Con}_{\omega}(X ; e, d)$ is a limit geodesic, not even in the particular case when $X$ is a finitely generated group with a word metric.

Example (of a group with continuously many non-limit geodesics in an asymptotic cone). On the two-dimensional unit sphere $\mathbb{S}^{2}$ consider a family of horizontal circles, in parallel planes, such that two consecutive circles are at spherical distance $\frac{\pi}{2^{k}}$, and such that the North and the South poles are at distance $\frac{\pi}{2^{k}}$ from two respective horizontal circles. Consider also a family of meridians of endpoints the North and the South poles, such that the intersection points of two consecutive meridians with the Equator are at spherical distance $\frac{\pi}{2^{k}}$.

The horizontal circles and the meridians compose a spherical grid $\Gamma_{k}^{\prime}$. We have that $\Gamma_{k}^{\prime} \subset \Gamma_{k+1}^{\prime}$. Let $\Gamma_{k}^{\prime \prime}$ be the graph obtained from $\Gamma_{k}^{\prime}$ by joining with spherical geodesics all pairs of vertices not on the same meridian nor on the same horizontal circle, and at distance at most $\frac{\pi}{\sqrt{2}^{k}}$. Let $\Gamma_{k}$ be the graph obtained from $\Gamma_{k}^{\prime \prime}$ by deleting all the vertical edges above the Equator, except the one having the East pole $(1,0,0)$ as an endpoint, and replacing each of them by a path of double length $\frac{\pi}{2^{k-1}}$ (see Figure 1). Let dist ${ }_{k}$ be the shortest-path metric on $\Gamma_{k}$.

Proposition 7.26 from [DS05b] applied to the sequence of graphs $\left(\Gamma_{k}\right.$, dist $\left._{k}\right)$, and Lemma 7.5 from the same paper imply that there exists a two-generated and recursively presented group $G$ with one asymptotic cone tree-graded, with all pieces isometric to $\mathbb{S}^{2}$. Moreover, from the construction of $G$ it follows that in each of the 


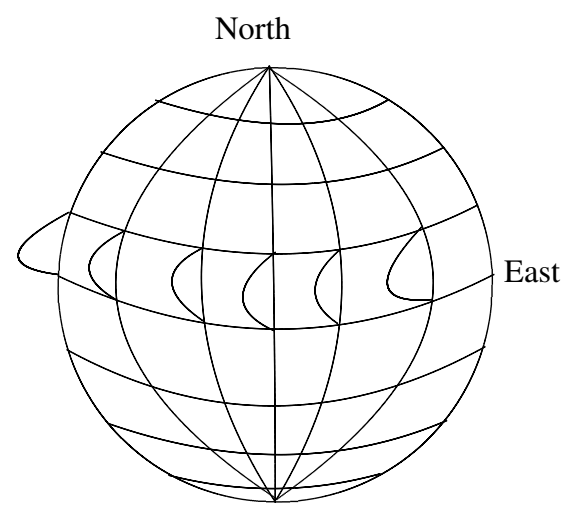

South

Figure 1. The graph $\Gamma_{k}$.

pieces, for an appropriate choice of the North, South and East poles, all geodesics joining North and South poles and not containing East pole are not limit geodesics.

The same argument as in [DS05b, §7] allows in fact to construct a two-generated and recursively presented group with continuously many non-homeomorphic asymptotic cones with the property that continuously many geodesics in each of these cones are not limit geodesics.

\section{Tree-graded metric spaces}

3.1. Definition and properties. The notion of tree-graded metric space has been introduced in [DS05b]. In this paper we use the following version of this notion. Recall that a subset $A$ in a geodesic metric space $X$ is called geodesic if every two points in $A$ can be joined by a geodesic contained in $A$.

Definition 3.1. Let $\mathbb{F}$ be a complete geodesic metric space and let $\mathcal{P}$ be a collection of closed geodesic subsets, called pieces. Suppose that the following two properties are satisfied:

$\left(T_{1}\right)$ Every two different pieces have at most one point in common.

$\left(T_{2}\right)$ Every simple non-trivial geodesic triangle in $\mathbb{F}$ is contained in one piece.

Then we say that the space $\mathbb{F}$ is tree-graded with respect to $\mathcal{P}$.

When there is no risk of confusion as to the set $\mathcal{P}$, we simply say that $\mathbb{F}$ is tree-graded. 
Remarks 3.2 (pieces need not cover the space). (1) In [DS05b] trivial geodesic triangles are allowed in property $\left(T_{2}\right)$. This is equivalent to asking that $\mathbb{F}$ is covered by the pieces in $\mathcal{P}$. In the present paper we remove this convention. The reason is that a main purpose when introducing the notion of tree-graded space is to produce a convenient notion of relatively hyperbolic metric space (called asymptotically treegraded metric space in [DS05b] and in this paper, see Definition 4.1). The condition that pieces cover $\mathbb{F}$ produces some unnatural restrictions for a space to be asymptotically tree-graded (i.e. relatively hyperbolic) with respect to a list of subsets. See Remark 4.12 for details.

(2) Possibly $\mathcal{P}$ is empty, in which case $\mathbb{F}$ is a real tree.

(3) When a group $G$ acts transitively on $\mathbb{F}$ (for instance when $\mathbb{F}$ is an asymptotic cone of a group) and $G$ permutes the pieces, the condition that pieces cover $\mathbb{F}$ is automatically satisfied.

All properties of tree-graded spaces in [DS05b, §2.1] hold with the new definition 3.1, as none of the proofs uses the property that pieces cover the space. In particular the results below hold. In what follows and throughout the paper by topological arc we mean a homeomorphic copy of the interval $[0,1]$.

Lemma 3.3 ([DS05b], §2.1). Let $x$ be an arbitrary point in $\mathbb{F}$ and let $T_{x}$ be the set of points $y \in \mathbb{F}$ which can be joined to $x$ by a topological arc intersecting every piece in at most one point.

The subset $T_{x}$ is a real tree and a closed subset of $\mathbb{F}$, and every topological arc joining two points in $T_{x}$ is contained in $T_{x}$. Moreover, for every $y \in T_{x}, T_{y}=T_{x}$.

Definition 3.4. A subset $T_{x}$ as in Lemma 3.3 is called a transversal tree in $\mathbb{F}$.

In [KKL98] is defined the notion of space of type I, which is equivalent to that of a tree-graded space with the extra property that for every $x$ the transversal tree $T_{x}$ is a geodesically complete tree which branches everywhere.

Remark 3.5. One can ensure that pieces in a tree-graded space cover it by adding to the list of pieces the transversal trees. Thus a tree-graded space $\mathbb{F}$ with set of pieces $\mathcal{P}$ in the sense of Definition 3.1 can be seen as tree-graded in the sense of Definition 2.1 in [DS05b] with respect to a set of pieces $\mathcal{P}^{\prime}$ such that $\mathcal{P}^{\prime} \backslash \mathcal{P}$ is a collection of real trees.

\subsection{Topological bigons contained in pieces}

Definition 3.6. Let $g_{1}$ and $g_{2}$ be topological arcs. A topological bigon (or $\mathcal{T}$-bigon, for short) formed by $g_{1}$ and $g_{2}$ is a union of a sub-arc $\mathfrak{g}_{1}^{\prime}$ of $g_{1}$ with a sub-arc $\mathfrak{g}_{2}^{\prime}$ of $\mathfrak{g}_{2}$ 
such that $\mathfrak{g}_{1}^{\prime}$ and $\mathfrak{g}_{2}^{\prime}$ have common endpoints $x$ and $y$. The endpoints of the $\mathcal{T}$-bigon are the points $x$ and $y$. The interior of the $\mathcal{T}$-bigon is the set $\mathrm{g}_{1}^{\prime} \cup \mathrm{g}_{2}^{\prime} \backslash\{x, y\}$.

If $\mathrm{g}_{1}^{\prime}$ and $\mathrm{g}_{2}^{\prime}$ intersect only in their endpoints then the $\mathcal{T}$-bigon is called simple (it is a simple loop).

Note that a $\mathcal{T}$-bigon with non-empty interior cannot be trivial, i.e. reduced to a point.

The results in this section are useful in arguments aiming to prove property $\left(T_{2}\right)$ for a collection of closed subsets of a metric space. In several contexts it proves necessary to deduce from $\left(T_{1}\right)$, and $\left(T_{2}\right)$ satisfied only for some special type of geodesic bigons, the general property $\left(T_{2}\right)$.

Lemma 3.7. Let $\mathrm{g}_{1}$ and $\mathrm{g}_{2}$ be two topological arcs with common endpoints. Then every point $z \in \mathfrak{g}_{1} \backslash \mathfrak{g}_{2}$ is in the interior of a simple $\mathcal{T}$-bigon formed by $\mathfrak{g}_{1}$ and $\mathfrak{g}_{2}$.

Proof. For $i=1,2, \mathfrak{g}_{i}:\left[0, \ell_{i}\right] \rightarrow Y$ is a topological embedding. Let $t \in\left[0, \ell_{1}\right]$ be such that $\mathrm{g}_{1}(t)=z$. The set $K=\mathfrak{g}_{1}^{-1}\left(\mathfrak{g}_{2}\left(\left[0, \ell_{2}\right]\right)\right)$ is a compact set not containing $t$. Let $r$ be the maximal element of the compact set $K \cap[0, t]$, and let $s$ be the minimal element of the compact set $K \cap\left[t, \ell_{1}\right]$. Then $\mathrm{g}_{1}(r)=\mathrm{g}_{2}\left(r^{\prime}\right)$ and $\mathrm{g}_{1}(s)=\mathrm{g}_{2}\left(s^{\prime}\right)$ for some $r^{\prime}, s^{\prime} \in\left[0, \ell_{2}\right]$. The union of $\mathrm{g}_{1}$ restricted to $[r, s]$ with $\mathrm{g}_{2}$ restricted to $\left[r^{\prime}, s^{\prime}\right]$ is a simple $\mathcal{T}$-bigon formed by $\mathfrak{g}_{1}$ and $\mathfrak{g}_{2}$, containing $z$ in its interior.

Lemma 3.8. Let $Y$ be a metric space and let $\mathcal{B}$ be a collection of subsets of $Y$ which satisfies property $\left(T_{1}\right)$.

Let $\mathrm{g}_{1}$ and $\mathrm{g}_{2}$ be two topological arcs with common endpoints and with the property that any non-trivial simple $\mathcal{T}$-bigon formed by $\mathrm{g}_{1}$ and $\mathrm{g}_{2}$ is contained in a subset in $\mathcal{B}$.

If $\mathrm{g}_{1}$ is contained in $B \in \mathscr{B}$ then $\mathrm{g}_{2}$ is contained in $B$.

Proof. Take $z$ an arbitrary point in $\mathfrak{g}_{2} \backslash \mathfrak{g}_{1}$. By Lemma 3.7 the point $z$ is in the interior of a simple $\mathcal{T}$-bigon formed by $\mathfrak{g}_{1}$ and $\mathfrak{g}_{2}$, of endpoints $z_{1}, z_{2}$. By hypothesis this $\mathcal{T}$-bigon is contained in a subset $B_{z} \in \mathscr{B}$. As $\left\{z_{1}, z_{2}\right\}$ is in $B \cap B_{z}$ it follows by $\left(T_{1}\right)$ that $B_{z}=B$ and that $z \in B$.

Proposition 3.9. Let $Y$ be a metric space and let $\mathscr{B}$ be a collection of closed subsets of $Y, \mathscr{B}$ with property $\left(T_{1}\right)$.

Let $\mathscr{L}_{1}$ and $\mathrm{g}_{1}$ be two topological arcs with common endpoints $u$, v. Let $\mathscr{L}_{2}$ and $\mathrm{g}_{2}$ be two, possibly identical, topological arcs with common endpoints $v, w$. Assume that:

(1) $\mathscr{L}_{1} \cap \mathscr{L}_{2}=\{v\}$;

(2) $\mathfrak{g}_{1} \cap \mathfrak{g}_{2}$ contains a point $a \neq v$; 
(3) all non-trivial simple $\mathcal{T}$-bigons formed either by $\mathfrak{g}_{1}$ and $\mathfrak{g}_{2}$, or by $\mathfrak{g}_{i}$ and $\mathscr{L}_{i}$, $i=1,2$, are contained in a subset in $\mathcal{B}$.

Then the $\mathcal{T}$-bigon formed by $\mathrm{g}_{1}$ and $\mathrm{g}_{2}$ with endpoints $a$ and $v$ is contained in a subset in $\mathcal{B}$.

Proof. Step 1. Let $\mathfrak{g}_{i}^{\prime}$ denote the sub-arc of $\mathfrak{g}_{i}$ of endpoints $a$ and $v, i=1,2$.

We prove that there exists $b \in \mathfrak{g}_{1}^{\prime} \cap \mathfrak{g}_{2}^{\prime} \backslash\{a\}$, such that the $\mathcal{T}$-bigon formed by $\mathfrak{g}_{1}^{\prime}$ and $\mathfrak{g}_{2}^{\prime}$ of endpoints $a, b$ is contained in some $B \in \mathscr{B}$.

Hypothesis (1) implies that either $a \notin \mathscr{L}_{1}$ or $a \notin \mathscr{L}_{2}$. Without loss of generality we may assume that $a \notin \mathscr{L}_{1}$. Then $a$ is in the interior of a simple $\mathcal{T}$-bigon formed by $\mathscr{L}_{1}$ and $\mathfrak{g}_{1}$, of endpoints $x$ and $y$, with $y$ on $\mathfrak{g}_{1}^{\prime}$. Property (3) implies that this $\mathcal{T}$-bigon is contained in a set $B_{1} \in \mathcal{B}$.

If $y \in \mathfrak{g}_{2}^{\prime}$ then take $b=y$.

Assume that $y \notin \mathfrak{g}_{2}^{\prime}$. Then $y$ is in the interior of a simple $\mathcal{T}$-bigon formed by $\mathfrak{g}_{1}^{\prime}$ and $\mathfrak{g}_{2}^{\prime}$, of endpoints $y_{1}, y_{2}$ (with $y_{2}$ closer to $v$ than $y_{1}$ on $\mathfrak{g}_{1}^{\prime}$ ). By (3) this $\mathcal{T}$-bigon is contained in some $B_{2} \in \mathscr{B}$ (see Figure 2). The intersection $B_{1} \cap B_{2}$ contains $\left\{y, y_{1}\right\}$ hence by $\left(T_{1}\right)$ we have that $B_{1}=B_{2}=B$. Take $b=y_{2}$.

The sub-arc of $\mathfrak{g}_{1}^{\prime}$ with endpoints $a$ and $b$ is contained in $B$. By property (3) we can apply Lemma 3.8 and obtain that the sub-arc of $\mathrm{g}_{2}^{\prime}$ in between $a$ and $b$ is also contained in $B$.

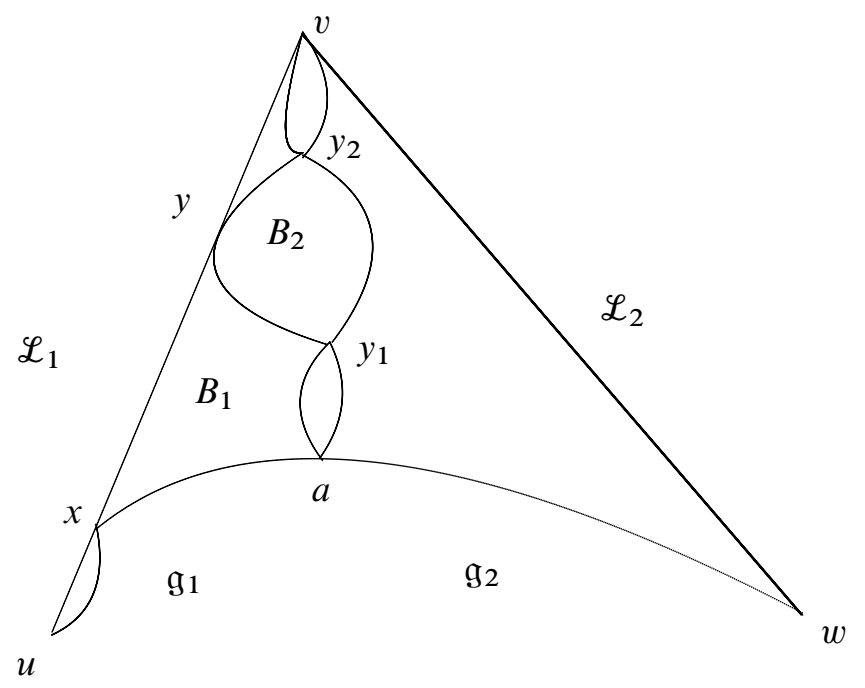

Figure 2. Step 1.

Step 2. Let $\mathcal{E}$ be the set of points $b \in \mathfrak{g}_{1}^{\prime} \cap \mathfrak{g}_{2}^{\prime} \backslash\{a\}$, such that the $\mathcal{T}$-bigon formed by $\mathfrak{g}_{1}^{\prime}$ and $\mathfrak{g}_{2}^{\prime}$ of endpoints $a, b$ is contained in some $B \in \mathscr{B}$. We prove that there exists $c \in \mathbb{E}$ such that $\mathfrak{g}_{1}$ between $c$ and $v$ contains no other point from $\mathcal{E}$. 
Note that by property $\left(T_{1}\right)$ of $\mathcal{B}$ all $\mathcal{T}$-bigons of endpoints $a$ and $b$, for some $b \in \mathcal{E}$, are contained in the same $B_{0} \in \mathcal{B}$.

Let $\varphi:[0, \ell] \rightarrow Y$ be a parametrization of $g_{1}, \varphi(\ell)=v$, and let $r$ be $\varphi^{-1}(a)$. The pre-image $\mathcal{E}^{\prime}=\varphi^{-1}(\mathcal{E})$ is contained in $(r, \ell]$. Let $T$ be the supremum of $\mathcal{E}^{\prime}$. Then $T=\lim t_{n}$ for some increasing sequence $\left(t_{n}\right)$ in $\mathcal{E}^{\prime}$, hence $c=\varphi(T)$ is the limit of the sequence of points $b_{n}=\varphi\left(t_{n}\right) \in \mathcal{E}$. Obviously $c \in \mathfrak{g}_{1}^{\prime} \cap \mathfrak{g}_{2}^{\prime} \backslash\{a\}$. Since $B_{0} \in \mathcal{B}$ is closed and $b_{n} \in B_{0}$, it follows that $c \in B_{0}$. Thus the sub-arc of $\mathfrak{g}_{1}^{\prime}$ between $a$ and $c$ is completely contained in $B_{0}$. By Lemma 3.8 and property (3), the $\mathcal{T}$-bigon formed by $\mathfrak{g}_{1}^{\prime}$ and $\mathfrak{g}_{2}^{\prime}$ of endpoints $a$ and $c$ is in $B_{0}$.

Step 3. We prove that the point $c$ obtained in Step 2 coincides with $v$.

Assume that $c \neq v$. Step 1 applied to the point $c$ instead of $a$ implies that there exists $d \in \mathfrak{g}_{1}^{\prime} \cap \mathfrak{g}_{2}^{\prime} \backslash\{c\}, d$ between $c$ and $v$ on both $\mathfrak{g}_{1}^{\prime}$ and $\mathfrak{g}_{2}^{\prime}$, such that the $\mathcal{T}$-bigon formed by $\mathfrak{g}_{1}^{\prime}$ and $\mathrm{g}_{2}^{\prime}$ of endpoints $c, d$ is contained in some $B^{\prime} \in \mathscr{B}$.

Since $c \neq v$ it cannot be contained simultaneously in $\mathscr{L}_{1}$ and in $\mathscr{L}_{2}$. Assume that $c \notin \mathscr{L}_{1}$. Then $c$ is in the interior of a simple $\mathcal{T}$-bigon formed by $g_{1}$ and $\mathscr{L}_{1}$. According to (3) this $\mathcal{T}$-bigon is contained in some $B^{\prime \prime} \in \mathscr{B}$. The intersections $B_{0} \cap B^{\prime \prime}$ and $B^{\prime} \cap B^{\prime \prime}$ both contain non-trivial sub-arcs of $\mathrm{g}_{1}^{\prime}$, therefore $B_{0}=B^{\prime \prime}=B^{\prime}$. Thus the point $d$ is in the set $\mathscr{E}$ and it is strictly between $c$ and $v$ on $\mathfrak{g}_{1}^{\prime}$. This contradicts the choice of $c$.

We conclude that $c=v$.

\section{Asymptotically tree-graded metric spaces}

4.1. Definitions and properties. Let $(X$, dist) be a geodesic metric space and let $\mathcal{A}=\left\{A_{i} \mid i \in I\right\}$ be a collection of subsets of $X$. In every asymptotic cone $\operatorname{Con}_{\omega}(X ; e, d)$, we consider the collection $\mathcal{A}_{\omega}$ of limit subsets

$$
\begin{aligned}
\left\{\lim _{\omega}\left(A_{i_{n}}\right) \mid i=\left(i_{n}\right)^{\omega} \in I^{\omega} \text { such that there exists an } M_{i}\right. \text { with } \\
\text { the property } \left.\operatorname{dist}\left(e_{n}, A_{i_{n}}\right) \leq{ }_{\omega} M_{i} d_{n}\right\} .
\end{aligned}
$$

Definition 4.1. The metric space $X$ is asymptotically tree-graded $(A T G)$ with respect to $\mathcal{A}$ if every asymptotic cone $\operatorname{Con}_{\omega}(X ; e, d)$ is tree-graded with respect to $\mathcal{A}_{\omega}$.

Following Convention 1.4, in the rest of the paper we shall assume that all ATG metric spaces are proper, that is, no subset $A \in \mathcal{A}$ contains $X$ in a tubular neighborhood of it.

The ATG property is meant to be an extension of the property of (strong) relative hyperbolicity from groups to metric spaces. Theorem 1.5 emphasizes that it is the correct property to work with. 
Remark 4.2. Let $X$ be ATG with respect to $\mathcal{A}=\left\{A_{i} \mid i \in I\right\}$.

(1) It is easy to see that for every $\tau>0$, the space $X$ is ATG with respect to $\left\{\mathcal{N}_{\tau}\left(A_{i}\right) \mid i \in I\right\}$.

(2) More generally, let $\mathscr{B}$ be a collection of subsets of $X$ such that there exists a constant $K \geq 0$ and a bijection $\phi: \mathcal{A} \rightarrow \mathscr{B}$ verifying $\operatorname{dist}_{H}(A, \phi(A)) \leq K$. Then $X$ is ATG with respect to $\mathscr{B}$.

The notion of ATG metric space can also be defined by a list of geometric conditions, without involving asymptotic cones. First we introduce some notation and terminology.

Notation 4.3. For a given quasi-geodesic $\mathfrak{p}$ and an $r>0$ we denote by $\breve{\mathfrak{p}}_{r}$ the set $\mathfrak{p} \backslash \mathcal{N}_{r}\left(\left\{\mathfrak{p}_{-}, \mathfrak{p}_{+}\right\}\right)$.

We say that a metric space $P$ is a geodesic (quasi-geodesic) $k$-gonal line if it is a union of $k$ geodesics (quasi-geodesics) $\mathfrak{q}_{1}, \ldots, \mathfrak{q}_{k}$ such that $\left(\mathfrak{q}_{i}\right)_{+}=\left(\mathfrak{q}_{i+1}\right)_{-}$for $i=1, \ldots, k-1$. If moreover $\left(\mathfrak{q}_{k}\right)_{+}=\left(\mathfrak{q}_{1}\right)_{-}$then we say that $P$ is a geodesic (quasi-geodesic) k-gon.

Let $P$ be a quasi-geodesic polygon, with set of vertices $\mathcal{V}$. Points in $P \backslash \mathcal{V}$ are called interior points of $P$.

Notation 4.4. Given a vertex $x \in \mathcal{V}$ and $\mathfrak{q}, \mathfrak{q}^{\prime}$ the consecutive edges of $P$ such that $x=\mathfrak{q}_{+}=\mathfrak{q}_{-}^{\prime}$, we denote the polygonal line $P \backslash\left(\mathfrak{q} \cup \mathfrak{q}^{\prime}\right)$ by $\mathcal{O}_{x}(P)$. When there is no possibility of confusion we simply denote it by $\mathcal{O}_{x}$.

Let $p \in P$. The inscribed radius in $p$ with respect to $P$ is either the distance from $p$ to the set $\mathcal{O}_{p}$, if $p$ is a vertex, or the distance from $p$ to the set $P \backslash q$ if $p$ is an interior point contained in the edge $q$ (see Figure 3, taken from [DS05b]).
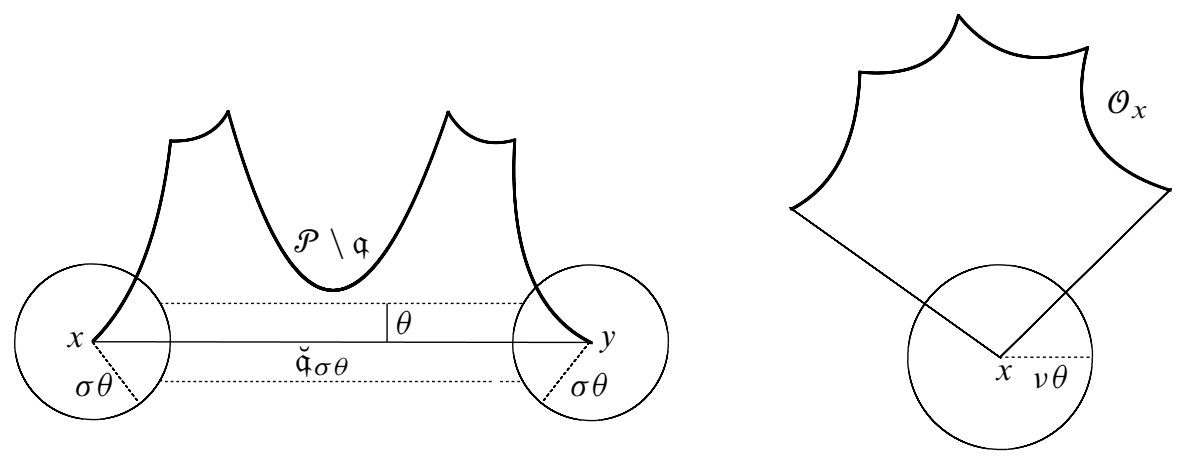

Figure 3. Properties $\left(\mathrm{Fat}_{1}\right)$ and $\left(\mathrm{Fat}_{2}\right)$. 
Definition 4.5 (fat polygon). Let $\theta>0, \sigma \geq 1$ and $v \geq 4 \sigma$. We call a $k$-gon $P$ with quasi-geodesic edges $(\theta, \sigma, v)$-fat if the following properties hold:

$\left(\mathrm{Fat}_{1}\right)$ (large inscribed radii in interior points, large comparison angles) for every edge $q$ we have, with the notation 4.3 , that

$$
\operatorname{dist}\left(\breve{\mathfrak{q}}_{\sigma \theta}, P \backslash \mathfrak{q}\right) \geq \theta \text {; }
$$

$\left(\mathrm{Fat}_{2}\right)$ (large inscribed radii in vertices, large edges) for every vertex $x$ we have that

$$
\operatorname{dist}\left(x, \mathcal{O}_{x}\right) \geq v \theta
$$

When $\sigma=2$ we say that $P$ is $(\theta, v)$-fat.

Lemma 4.6. Let $P$ be a polygon $(\theta, \sigma, v)$-fat for some $\theta>0, \sigma \geq 1$ and $v \geq 4 \sigma$. Then any two edges of $P$ without a common vertex are at distance at least $\theta$ from each other.

Proof. Let $\mathfrak{q}$ and $\mathfrak{q}^{\prime}$ be two edges without a common vertex. Assume that there exists a point $a \in \mathfrak{q}$ such that $\operatorname{dist}\left(a, \mathfrak{q}^{\prime}\right)<\theta$. Property (Fat $\left.{ }_{1}\right)$ implies that $a \in \mathcal{N}_{\sigma \theta}(\{x, y\})$, where $x, y$ are the endpoints of $\mathfrak{q}$. Property $\left(\mathrm{Fat}_{2}\right)$ implies that dist $\left(\{x, y\}, \mathfrak{q}^{\prime}\right) \geq v \theta$. Therefore $\operatorname{dist}\left(a, \mathfrak{q}^{\prime}\right) \geq(v-\sigma) \theta \geq 3 \sigma \theta \geq 3 \theta$. This contradicts the assumption that $\operatorname{dist}\left(a, \mathfrak{q}^{\prime}\right)<\theta$.

The following lemma describes a situation in which given two consecutive edges of a geodesic polygon, any two points on each of these edges which are at distance at least $2 \theta$ from the common vertex are at distance at least $\theta$ from one another.

Lemma 4.7. Let $P$ be a geodesic polygon with two consecutive edges $[x, y]$ and $[y, z]$ such that $\operatorname{dist}(x,[y, z])=\operatorname{dist}(x, y)$. Then both the distance from $[x, y] \backslash B(y, 2 \theta)$ to $[y, z]$, and the distance from $[y, z] \backslash B(y, 2 \theta)$ to $[x, y]$ are at least $\theta$.

Proof. The distance from $[x, y] \backslash B(y, 2 \theta)$ to $[y, z]$ is $2 \theta$ because of the hypothesis that $\operatorname{dist}(x,[y, z])=\operatorname{dist}(x, y)$.

Now assume that there exists $p \in[y, z] \backslash B(y, 2 \theta)$ and $p^{\prime} \in[x, y]$ such that $\operatorname{dist}\left(p, p^{\prime}\right)<\theta$. Then $\operatorname{dist}\left(y, p^{\prime}\right) \geq \operatorname{dist}(y, p)-\operatorname{dist}\left(p, p^{\prime}\right)>\theta>\operatorname{dist}\left(p, p^{\prime}\right)$. It follows that $\operatorname{dist}(x, p) \leq \operatorname{dist}\left(x, p^{\prime}\right)+\operatorname{dist}\left(p^{\prime}, p\right)<\operatorname{dist}\left(x, p^{\prime}\right)+\operatorname{dist}\left(p^{\prime}, y\right)=$ $\operatorname{dist}(x, y)$. This contradicts the fact that $\operatorname{dist}(x,[y, z])=\operatorname{dist}(x, y)$.

We shall also need in the sequel a way of obtaining from a fat $k$-gon a fat $(k+1)$ gon. This is described in the next lemma. 
Lemma 4.8. Let $P$ be a geodesic $k$-gon with two consecutive edges $[x, y]$ and $[y, z]$, such that $\operatorname{dist}(x,[y, z])=\operatorname{dist}(x, y)$. If $P$ is $(\theta, v)$-fat then the $(k+1)$-gon $P^{\prime}$ obtained from $P$ by adding as a vertex the point $v \in[x, y]$ with $\operatorname{dist}(v, y)=\frac{v \theta}{2}$ is $\left(\theta, \frac{v}{2}\right)$-fat.

Proof. Property $\left(\mathrm{Fat}_{1}\right)$ for $P^{\prime}$ follows easily from property (Fat $\left.{ }_{1}\right)$ for $P$.

Property $\left(\mathrm{Fat}_{2}\right)$ holds for all the vertices different from $x, v, y$, by property $\left(\mathrm{Fat}_{2}\right)$ in $P$.

The polygonal line $\mathcal{O}_{x}\left(P^{\prime}\right)=\mathcal{O}_{x}(P) \cup[v, y]$ is in the $\frac{v \theta}{2}$-tubular neighborhood of $\mathcal{O}_{x}(P)$, hence at distance at least $\frac{v \theta}{2}$ from $x$.

The polygonal line $\mathcal{O}_{y}\left(P^{\prime}\right)$ is equal to $\mathcal{O}_{y}(P) \cup[x, v]$. The line $\mathcal{O}_{y}(P)$ is at distance $\geq v \theta$ from $y$ and $[x, v]$ is at distance $\frac{v \theta}{2}$ from $y$.

Finally, $\mathcal{O}_{v}\left(P^{\prime}\right)=\mathcal{O}_{y}(P) \cup[y, z]$. Since $\operatorname{dist}(v, y)=\frac{v \theta}{2}$ it follows that $\operatorname{dist}\left(v, \mathcal{O}_{y}\right) \geq \frac{v \theta}{2}$. If there exists $p \in[y, z]$ such that $\operatorname{dist}(v, p)<\frac{v \theta}{2}$ then $\operatorname{dist}(x, p) \leq \operatorname{dist}(x, v)+\operatorname{dist}(v, p)<\operatorname{dist}(x, v)+\frac{v \theta}{2}=\operatorname{dist}(x, y)$. This contradicts the hypothesis that $\operatorname{dist}(x,[y, z])=\operatorname{dist}(x, y)$.

A metric space $X$ can be defined to be asymptotically tree-graded with respect to a collection of subsets $\mathcal{A}$ without using asymptotic cones, simply by putting conditions on intersections of tubular neighborhoods of subsets in $\mathcal{A}$, and on the behavior of geodesics and of fat polygons with respect to such neighborhoods.

Theorem 4.9 ([DS05b], Theorem 4.1 and Remark 4.2 (3)). Let (X, dist) be a geodesic metric space and let $\mathcal{A}=\left\{A_{i} \mid i \in I\right\}$ be a collection of subsets of $X$. The metric space $X$ is asymptotically tree-graded with respect to $\mathcal{A}$ if and only if the following properties are satisfied:

$\left(\alpha_{1}\right)$ For every $\delta>0$ the diameters of the intersections $\mathcal{N}_{\delta}\left(A_{i}\right) \cap \mathcal{N}_{\delta}\left(A_{j}\right)$ are uniformly bounded for all $i \neq j$.

$\left(\alpha_{2}\right)$ There exists $\varepsilon$ in $\left[0, \frac{1}{2}\right)$ and $M>0$ such that for every geodesic $g$ of length $\ell$ and every $A \in \mathcal{A}$ with $\mathfrak{g}(0), \mathfrak{g}(\ell) \in \mathcal{N}_{\varepsilon \ell}(A)$ we have that $\mathfrak{g}([0, \ell]) \cap \mathcal{N}_{M}(A) \neq \emptyset$.

$\left(\alpha_{3}\right)$ For every $k \geq 2$ there exist $\theta>0, v \geq 8$ and $\chi>0$ such that every $k$-gon $P$ in $X$ with geodesic edges which is $(\theta, v)$-fat satisfies $P \subset \mathcal{N}_{\chi}(A)$ for some $A \in \mathcal{A}$.

Remarks 4.10 ([DS05b], Theorem 4.1 and Remark 4.2). (1) Property $\left(\alpha_{2}\right)$ from Theorem 4.9 is a slight modification of the similar property appearing in Theorem 4.1 in [DS05b]. Nevertheless it implies property $\left(\alpha_{2}^{\varepsilon}\right)$ from [DS05b, Remark 4.2, (3)], which accounts for the accuracy of the modified statement.

(2) As a necessary condition, $\left(\alpha_{2}\right)$ can be strengthened to "for every $\varepsilon$ from $\left[0, \frac{1}{2}\right)$ there exists $M>0$ such that etc." 
Notation 4.11. We denote by $\operatorname{diam}_{\delta}$ a uniform bound provided by property $\left(\alpha_{1}\right)$ for an arbitrary $\delta \geq 0$.

Remarks 4.12 (on the condition that pieces cover the space). (1) In property $\left(T_{2}\right)$ of the definition of a tree-graded space (Definition 3.1), we might allow for trivial geodesic triangles, that is, we might ask that pieces cover the space. This would also change the definition of an asymptotically tree-graded space; in Theorem 4.9 in order for the equivalence to hold the following condition would have to be added:

$\left(\alpha_{0}\right)$ there exists $\tau \geq 0$ such that $X=\bigcup_{A \in \mathcal{A}} \mathcal{N}_{\tau}(A)$.

The difference between these definitions of (asymptotically) tree-graded spaces and the ones that are actually used in the paper consists in the addition of a set of singletons to the collection of pieces $\mathcal{P}$ (respectively, to the collection of subsets $\mathcal{A}$ ). Indeed, if $(X, \mathcal{A})$ satisfy only $\left(\alpha_{1}\right),\left(\alpha_{2}\right),\left(\alpha_{3}\right)$ but not $\left(\alpha_{0}\right)$ then it suffices to add some singletons to $\mathcal{A}$ in order to ensure $\left(\alpha_{0}\right)$. For some $\tau>0$ consider in $X \backslash \bigcup_{A \in \mathcal{A}} \mathcal{N}_{\tau}(A)$ a maximal subset $\wp$ with the property that $\operatorname{dist}\left(p, p^{\prime}\right) \geq \tau$ for every $p, p^{\prime} \in \wp$. The space $X$ coincides with $\bigcup_{A \in \mathcal{A}} \mathcal{N}_{\tau}(A) \cup \bigcup_{p \in \wp} \mathcal{N}_{\tau}(\{p\})$. Properties $\left(\alpha_{1}\right)$ and $\left(\alpha_{2}\right)$ are obviously satisfied by singletons, whence $X$ is ATG with respect to $\mathcal{A}^{\prime}=$ $\mathcal{A} \cup\{\{p\} \mid p \in \wp\}$; moreover $\mathcal{A}^{\prime}$ also satisfies $\left(\alpha_{0}\right)$.

(2) Let $\mathbb{H}^{3}$ be the 3-dimensional real hyperbolic space and let $\left(\mathrm{Hbo}_{n}\right)_{n \in \mathbb{N}}$ be a countable collection of pairwise disjoint open horoballs. The complementary set $X_{0}=X \backslash \bigsqcup_{n \in \mathbb{N}} \mathrm{Hbo}_{n}$ and the collection of boundary horospheres $\mathcal{A}=$ $\left\{\partial \mathrm{Hbo}_{n} \mid n \in \mathbb{N}\right\}$ is the typical example one has in mind when trying to define relative hyperbolicity for metric spaces. The pair $\left(X_{0}, \mathcal{A}\right)$ does not in general satisfy $\left(\alpha_{0}\right)$, one has to add singletons to $\mathcal{A}$ to ensure that property. In order to remove this inconvenient, we have given up the condition that pieces cover the space in the Definition 3.1 of tree-graded spaces.

Remark 4.13. If $X$ is a metric space ATG with respect to $\mathcal{A}$, and a group $G$ acts $\mathcal{K}$-transitively (in the sense of Definition 2.1 , with $\mathcal{K} \geq 0$ ) by isometries on $X, G$ permuting the subsets in $\mathcal{A}$, then property $\left(\alpha_{0}\right)$ is satisfied with $\tau=\mathcal{K}$.

It is for instance the case when $X$ is itself a group and $\mathcal{A}$ is the collection of left cosets of a family of subgroups.

4.2. Property $\left(\boldsymbol{T}_{2}\right)$ and polygons with limit edges. Property $\left(\alpha_{3}\right)$ in the definition of a metric space $X$ ATG with respect to a collection $\mathcal{A}$ (requiring that fat polygons stay in tubular neighborhoods of subsets in $\mathcal{A}$ ) is used to prove property $\left(T_{2}\right)$ in an arbitrary asymptotic cone of $X$ with respect to the collection of limit sets $\mathcal{A}_{\omega}$ (requiring that simple non-trivial geodesic triangles are contained in pieces from $\mathcal{A}_{\omega}$ ). If $X$ is such that any geodesic in an asymptotic cone of it is a limit geodesic (for instance if $X$ is a $\mathrm{CAT}(0)$ metric space) then it suffices to have property $\left(\alpha_{3}\right)$ for fat hexagons, that is: 
$\left(\beta_{3}\right)$ there exists $\theta>0, v \geq 8$ and $\chi>0$ such that any geodesic hexagon $(\theta, v)$-fat is contained in $\mathcal{N}_{\chi}(A)$, for some $A \in \mathcal{A}$.

This is due to the following general fact.

Proposition 4.14. Let ( $X$, dist) be a geodesic metric space and let $\theta>0$ and $v \geq 8$ be two arbitrary constants. In any asymptotic cone $\operatorname{Con}_{\omega}(X ; e, d)$, any simple nontrivial triangle whose edges are limit geodesics is the limit set $\lim _{\omega}\left(H_{n}\right)$ of a sequence $\left(H_{n}\right)$ of geodesic hexagons that are $(\theta, v)$-fat $\omega$-almost surely.

Proof. Consider a non-trivial simple geodesic triangle $\Delta$ in an asymptotic cone $\operatorname{Con}_{\omega}(X ; e, d)$, whose edges $[a, b],[b, c]$ and $[c, a]$ appear as limit sets of sequences $\left[a_{n}, b_{n}^{\prime}\right],\left[b_{n}, c_{n}^{\prime}\right]$ and $\left[c_{n}, a_{n}^{\prime}\right]$ of geodesics in $X$. We have that $\operatorname{dist}\left(a_{n}, a_{n}^{\prime}\right), \operatorname{dist}\left(b_{n}, b_{n}^{\prime}\right)$ and $\operatorname{dist}\left(c_{n}, c_{n}^{\prime}\right)$ are of order $o\left(d_{n}\right) \omega$-almost surely.

Let $d_{n}^{A}$ be the maximum between $\operatorname{dist}\left(\left[a_{n}, b_{n}^{\prime}\right],\left[a_{n}^{\prime}, c_{n}\right]\right)$ and $v \theta$. Note that $d_{n}^{A}>0$ and that $d_{n}^{A}={ }_{\omega} o\left(d_{n}\right)$. Take $a_{n}^{1}$ to be the farthest from $a_{n}$ point on $\left[a_{n}, b_{n}^{\prime}\right]$ at distance $d_{n}^{A}$ from $\left[a_{n}^{\prime}, c_{n}\right]$. Consider then $a_{n}^{2}$ the farthest from $a_{n}^{\prime}$ point on $\left[a_{n}^{\prime}, c_{n}\right]$ at distance $d_{n}^{A}$ from $a_{n}^{1}$. Obviously $\operatorname{dist}\left(a_{n}^{1}, a_{n}^{2}\right)=d_{n}^{A}$.

The pairs of points $\left(b_{n}^{1}, b_{n}^{2}\right)$ in $\left[b_{n}, c_{n}^{\prime}\right] \times\left[b_{n}^{\prime}, a_{n}\right]$, and respectively $\left(c_{n}^{1}, c_{n}^{2}\right)$ in $\left[c_{n}, a_{n}^{\prime}\right] \times\left[c_{n}^{\prime}, b_{n}\right]$ are chosen similarly. Since the limit triangle $\Delta$ is simple, it follows that the sets $\left\{a_{n}, a_{n}^{\prime}, a_{n}^{1}, a_{n}^{2}\right\},\left\{b_{n}, b_{n}^{\prime}, b_{n}^{1}, b_{n}^{2}\right\}$ and $\left\{c_{n}, c_{n}^{\prime}, c_{n}^{1}, c_{n}^{2}\right\}$ have $\omega$-almost surely diameters of order $o\left(d_{n}\right)$. Hence the sequence of geodesic hexagons $H_{n}$ of vertices $a_{n}^{1}, b_{n}^{2}, b_{n}^{1}, c_{n}^{2}, c_{n}^{1}, a_{n}^{2}$ with edges $\left[a_{n}^{1}, b_{n}^{2}\right] \subset\left[a_{n}, b_{n}^{\prime}\right],\left[b_{n}^{1}, c_{n}^{2}\right] \subset\left[b_{n}, c_{n}^{\prime}\right]$, $\left[c_{n}^{1}, a_{n}^{2}\right] \subset\left[c_{n}, a_{n}^{\prime}\right]$, has the property that $\lim _{\omega}\left(H_{n}\right)$ is $\Delta$. It remains to prove that $H_{n}$ is $\omega$-almost surely $(\theta, v)$-fat.

$\left(\mathrm{Fat}_{1}\right)$ : The fact that the edge $\left[a_{n}^{1}, a_{n}^{2}\right]$ is at distance $O\left(d_{n}\right)$ from $\left[b_{n}^{2}, b_{n}^{1}\right] \cup\left[b_{n}^{1}, c_{n}^{2}\right] \cup$ $\left[c_{n}^{2}, c_{n}^{1}\right]$ and Lemma 4.7 imply that $\left[a_{n}^{1}, a_{n}^{2}\right]$ satisfies property (Fat $\left.{ }_{1}\right)$.

In the same manner it can be shown that the edges $\left[b_{n}^{1}, b_{n}^{2}\right]$ and $\left[c_{n}^{1}, c_{n}^{2}\right]$ satisfy $\left(\right.$ Fat $\left._{1}\right)$.

The edge $\left[a_{n}^{1}, b_{n}^{2}\right]$ is at distance $O\left(d_{n}\right)$ from $\left[c_{n}^{1}, c_{n}^{2}\right]$. The choice of $a_{n}^{1}$ and of the pair $\left(b_{n}^{1}, b_{n}^{2}\right)$ implies that $\left[a_{n}^{1}, b_{n}^{2}\right]$ is at distance at least $\nu \theta$ from $\left[b_{n}^{1}, c_{n}^{2}\right] \cup\left[c_{n}^{1}, a_{n}^{2}\right]$. Lemma 4.7 allows to conclude that $\left[a_{n}^{1}, b_{n}^{2}\right]$ satisfies $\left(\mathrm{Fat}_{1}\right)$.

Similar arguments show that the edges $\left[b_{n}^{1}, c_{n}^{2}\right]$ and $\left[c_{n}^{1}, a_{n}^{2}\right]$ satisfy (Fat $\left.{ }_{1}\right)$.

$\left(\mathrm{Fat}_{2}\right)$ : The distance from $a_{n}^{1}$ to $\left[a_{n}^{2}, c_{n}^{1}\right]$ is at least $v \theta$ by the choice if $a_{n}^{1}$, while the distance to $\left[b_{n}^{2}, b_{n}^{1}\right] \cup\left[b_{n}^{1}, c_{n}^{2}\right] \cup\left[c_{n}^{2}, c_{n}^{1}\right]$ is $O\left(d_{n}\right)$. The same kind of argument shows that $\left(\mathrm{Fat}_{2}\right)$ is satisfied $\omega$-almost surely by all the vertices of $H_{n}$.

In general not every geodesic in an asymptotic cone is a limit geodesic (see the example in the end of Section 2.2). Thus, in order to ensure that in every asymptotic cone every non-trivial simple geodesic triangle is contained in some limit set from $\mathcal{A}_{\omega}$ (i.e. property $\left(T_{2}\right)$ ), in [DS05b] property $\left(\alpha_{3}\right)$ is required for all fat polygons, not 
just for hexagons, and it is combined with the fact that limit sets are closed, and with the following result.

Lemma 4.15 ([DS05b], Proposition 3.34). Let $\Delta$ be an arbitrary simple geodesic triangle in $\operatorname{Con}_{\omega}(X ; e, d)$. For every $\varepsilon>0$ sufficiently small there exists $k_{0}=k_{0}(\varepsilon)$ and a simple geodesic triangle $\Delta_{\varepsilon}$ with the following properties:

(a) $\operatorname{dist}_{H}\left(\Delta, \Delta_{\varepsilon}\right) \leq \varepsilon$;

(b) $\Delta_{\varepsilon}$ contains the midpoints of the edges of $\Delta$;

(c) for every $\theta>0$ and $v \geq 8$ the triangle $\Delta_{\varepsilon}$ is the limit set $\lim _{\omega}\left(P_{n}^{\varepsilon}\right)$ of a sequence $\left(P_{n}^{\varepsilon}\right)$ of geodesic $k$-gons in $X$, for some $k \leq k_{0}$, that are $(\theta, v)$-fat $\omega$-almost surely.

Remark 4.16. If $\Delta$ is non-trivial then the set of midpoints of edges of $\Delta$ has cardinality 3 , hence the triangles $\Delta_{\varepsilon}$ are also non-trivial.

In this section we prove that if in every asymptotic cone property $\left(T_{1}\right)$ holds for the collection of limit sets $\mathcal{A}_{\omega}$ (that is, two distinct sets in $\mathcal{A}_{\omega}$ intersect in at most one point), then property $\left(\beta_{3}\right)$ for the collection $\mathcal{A}$, granting that fat hexagons stay in tubular neighborhoods of subsets in $\mathcal{A}$, suffices to deduce property $\left(T_{2}\right)$ for $\mathcal{A}_{\omega}$ (i.e. that every simple non-trivial geodesic triangle is contained in a set from $\left.\mathcal{A}_{\omega}\right)$. To this end, we define the following property in an asymptotic cone $\operatorname{Con}_{\omega}(X ; e, d)$ :

$\left(\Pi_{k}\right)$ every simple non-trivial $k$-gon with edges limit geodesics is contained in a subset from $\mathcal{A}_{\omega}$.

Corollary 4.17. Assume that in an asymptotic cone $\operatorname{Con}_{\omega}(X ; e, d)$ a collection $\mathcal{A}_{\omega}$ of closed subsets satisfies properties $\left(T_{1}\right)$ and $\left(\Pi_{k}\right)$ for every $k \in \mathbb{N}, k \geq 3$. Then $\mathcal{A}_{\omega}$ satisfies $\left(T_{2}\right)$.

Proof. Consider a simple non-trivial geodesic triangle $\Delta$ in $\operatorname{Con}_{\omega}(X ; e, d)$. By Lemma 4.15 for every large enough $k \in \mathbb{N}$ there exists a simple non-trivial geodesic triangle $\Delta_{k}$ at Hausdorff distance at most $\frac{1}{k}$ from $\Delta$, containing the midpoints of the edges of $\Delta$, moreover $\Delta_{k}=\lim _{\omega}\left(P_{n}^{(k)}\right)$, where $P_{n}^{(k)}$ is $n$ - $\omega$-almost surely a geodesic $m$-gon, $m=m(k)$. By property $\left(\Pi_{m}\right)$ the triangle $\Delta_{k}$ is contained in some $A_{k} \in \mathcal{A}_{\omega}$. All $A_{k}$ contain the midpoints of the edges of $\Delta$. Property $\left(T_{1}\right)$ implies that there exists $A \in \mathcal{A}_{\omega}$ such that $A_{k}=A$ for all $k$. All $\Delta_{k}$ are in $A, \Delta$ is the limit of $\Delta_{k}$ with respect to the Hausdorff distance, and $A$ is closed, therefore $\Delta \subset A$.

In view of Corollary 4.17 it suffices to prove that $\mathcal{A}_{\omega}$ satisfies $\left(\Pi_{k}\right)$ for all $k \geq 3$ to deduce that $\mathcal{A}_{\omega}$ satisfies property $\left(T_{2}\right)$.

Obviously $\left(\Pi_{k}\right)$ implies $\left(\Pi_{i}\right)$ for every $i<k$. It turns out that with the additional assumption that $\left(T_{1}\right)$ is satisfied, the converse implication also holds. 
Lemma 4.18. Assume that in an asymptotic cone $\operatorname{Con}_{\omega}(X ; e, d)$, the collection of subsets $\mathcal{A}_{\omega}$ satisfies the properties $\left(T_{1}\right)$ and $\left(\Pi_{3}\right)$. Then $\mathcal{A}_{\omega}$ satisfies property $\left(\Pi_{k}\right)$ for every $k \geq 3$.

Proof. We prove property $\left(\Pi_{k}\right)$ by induction on $k$. The cases $k=2$ and $k=3$ hold by hypothesis. Assume that the statement is true for every $k \leq m-1$ and consider a simple non-trivial geodesic $m$-gon $P$ in $\operatorname{Con}_{\omega}(X ; e, d), m \geq 4$, with edges limit geodesics.

Let $[x, y]$ and $[y, z]$ be two consecutive edges of $P$, in clockwise order. Denote by $\mathscr{L}_{1}$ the union of the two edges $[x, y] \cup[y, z]$ of $P$, and by $\mathscr{L}$ the union of the other $m-2$ edges of $P$, in clockwise order.

Consider a limit geodesic $\mathrm{g}$ joining $x$ and $z$. If $\mathrm{g}$ coincides with $\mathscr{L}_{1}$ or with $\mathscr{L}$ then $P$ is a simple geodesic polygon with at most $m-1$ edges, all of them limit geodesics. By the inductive hypothesis $P$ is contained in a subset $A$ in $\mathcal{A}_{\omega}$.

Assume that $g$ does not coincide either with $\mathscr{L}_{1}$ or with $\mathscr{L}$.

Step 1 . We prove that $\mathrm{g} \cup \mathscr{L}$ is contained in some $A \in \mathcal{A}_{\omega}$.

Let $\alpha \in \mathscr{L} \backslash \mathrm{g}$. Lemma 3.7 implies that $\alpha$ is in the interior of a simple $\mathcal{T}$-bigon formed by $\mathscr{L}$ and $\mathfrak{g}$, of endpoints $a$ and $b$, with $a$ closer to $x$ than $b$ on $\mathscr{L}$. This $\mathcal{T}$-bigon is a geodesic polygon with at most $m-1$ edges which are limit geodesics, therefore by the inductive hypothesis it is contained in a subset $A \in \mathcal{A}_{\omega}$.

If $a=x$ and $b=z$ then $\mathfrak{g} \cup \mathscr{L}$ is a simple $\mathcal{T}$-bigon and it is contained in some $A \in \mathcal{A}_{\omega}$ by the inductive hypothesis. Assume therefore that $(a, b) \neq(x, z)$. Without loss of generality we may assume that $a \neq x$.

We apply Proposition 3.9 to $\mathscr{L}_{1}, \mathrm{~g}_{1}=\mathrm{g}$, and $\mathrm{g}_{2}=\mathscr{L}_{2}$ the sub-arc of $\mathscr{L}$ in between $x$ and $\alpha$. Property (3) is satisfied by the hypothesis of the induction. It follows that the $\mathcal{T}$-bigon formed by $g$ and $\mathscr{L}$ of endpoints $x$ and $a$ is contained in some $A_{1} \in \mathcal{A}_{\omega}$.

The point $a$ is in $\mathscr{L} \backslash \mathscr{L}_{1}$, hence it is in $\mathfrak{g} \backslash \mathscr{L}_{1}$. By Lemma 3.7, $a$ is in the interior of some simple $\mathcal{T}$-bigon formed by $\mathscr{L}_{1}$ and $\mathfrak{g}$, and by $\left(\Pi_{3}\right)$ this $\mathcal{T}$-bigon is in a subset $A_{1}^{\prime} \in \mathcal{A}_{\omega}$. Since $A_{1}^{\prime} \cap A$ and $A_{1}^{\prime} \cap A_{1}$ contain non-trivial sub-arcs of g property $\left(T_{1}\right)$ implies that $A=A_{1}^{\prime}=A_{1}$.

If moreover $b \neq z$, a similar argument gives that the $\mathcal{T}$-bigon formed by $\mathrm{g}$ and $\mathscr{L}$ of endpoints $z$ and $b$ is contained in $A$ (see Figure 4).

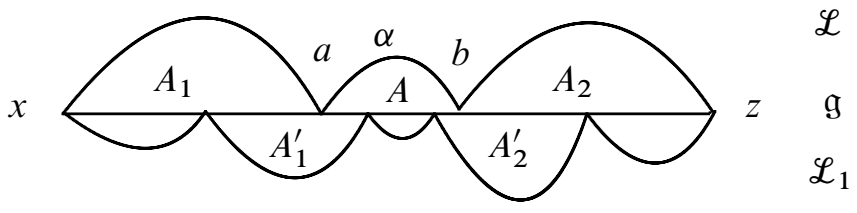

Figure 4. Step 1 in the proof of Lemma 4.18. 
We conclude that $\mathrm{g} \cup \mathscr{L}$ is contained in $A$.

Step 2. We prove that $\mathscr{L}_{1}$ is also contained in $A$. Property $\left(\Pi_{3}\right)$ implies that any non-trivial simple $\mathcal{T}$-bigon formed by $\mathscr{L}_{1}$ and $\mathrm{g}$ is contained in a subset in $\mathcal{A}_{\omega}$. We apply Lemma 3.8 to $\mathscr{L}_{1}$ and $\mathrm{g} \subset A$ and we conclude that $\mathscr{L}_{1} \subset A$. Consequently $A$ contains $\mathscr{L}_{1} \cup \mathscr{L}=P$.

Corollary 4.19. Assume that in an asymptotic cone $\operatorname{Con}_{\omega}(X ; e, d)$, the collection of closed subsets $\mathcal{A}_{\omega}$ satisfies properties $\left(T_{1}\right)$ (two distinct subsets from $\mathcal{A}_{\omega}$ intersect in at most one point) and $\left(\Pi_{3}\right)$ (simple non-trivial triangles with edges limit geodesics are contained in subsets from $\left.\mathcal{A}_{\omega}\right)$. Then $\mathcal{A}_{\omega}$ satisfies property $\left(T_{2}\right)$ (i.e., all simple non-trivial geodesic triangles are contained in subsets from $\left.\mathcal{A}_{\omega}\right)$.

Corollary 4.20. Let $X$ be a geodesic metric space and $\mathcal{A}$ a collection of subsets in $X$, such that property $\left(\beta_{3}\right)$ is satisfied (i.e., fat hexagons are contained in tubular neighborhoods of subsets from $\mathcal{A})$, and such that in any asymptotic cone $\operatorname{Con}_{\omega}(X ; e, d)$, the collection of limit subsets $\mathcal{A}_{\omega}$ satisfies property $\left(T_{1}\right)$. Then $\mathcal{A}_{\omega}$ satisfies prop$\operatorname{erty}\left(T_{2}\right)$.

Note that the only thing missing in Corollary 4.20 to conclude that $X$ is ATG with respect to $\mathcal{A}$ is that $\mathcal{A}_{\omega}$ is composed of geodesic subsets.

Another useful consequence of Proposition 4.14 is the following.

Corollary 4.21. Let (X, dist) be a geodesic metric space. Assume that for some $\theta>0$ and $v \geq 8$ the set of $(\theta, v)$-fat geodesic hexagons is either empty or composed of hexagons of uniformly bounded diameter. Then $X$ is hyperbolic.

Proof. Proposition 4.14 implies that in any asymptotic cone of $X$ any simple triangle with edges limit geodesics is trivial. This statement can be extended by induction to all polygons. Indeed, suppose that in any asymptotic cone of $X$ for all $3 \leq k \leq m-1$ all simple $k$-gons with edges limit geodesics are trivial. Consider $P$ a simple $m$-gon with edges limit geodesics in some $\operatorname{Con}_{\omega}(X ; e, d)$. Let $[x, y]$ and $[y, z]$ be two consecutive edges of $P$ and let $\mathrm{g}$ be a limit geodesic joining $x$ and $z$. All simple $\mathcal{T}$-bigons formed by $[x, y] \cup[y, z]$ and $g$ must be trivial by the inductive hypothesis, thus $\mathrm{g}=[x, y] \cup[y, z]$. It follows that $P$ is a simple $(m-1)$-gon with edges limit geodesics, hence by the inductive hypothesis it is trivial.

Lemma 4.15 and Remark 4.16 imply that in any $\operatorname{Con}_{\omega}(X ; e, d)$ any simple geodesic triangle must be trivial. It follows that $\operatorname{Con}_{\omega}(X ; e, d)$ is a real tree, and since this holds for all asymptotic cones we conclude that $X$ is hyperbolic ([Gro93, $\S 2 . A]$, see also [Dru02, §3]).

4.3. New definitions, useful for the rigidity of relatively hyperbolic groups. In this section new versions of the definition of an ATG metric space are stated and 
proved. They play an important part later on, in the proof of the quasi-isometric invariance of relative hyperbolicity.

Theorem 4.22. In Theorem 4.9 the following modifications can be made in the list of properties defining an asymptotically tree-graded metric space:

(Modif $\left.{ }_{1}\right)$ property $\left(\alpha_{3}\right)$ requiring that fat polygons stay in tubular neighborhoods of subsets in $\mathcal{A}$ can be replaced by property $\left(\beta_{3}\right)$ requiring the same thing but only for hexagons;

(Modif $)_{2}$ property $\left(\alpha_{2}\right)$ can be either maintained or replaced by one of the following two properties:

$\left(\beta_{2}\right)$ there exists $\epsilon>0$ and $M \geq 0$ such that for any geodesic $g$ of length $\ell$ and any $A \in \mathcal{A}$ satisfying $\mathfrak{g}(0), \mathfrak{g}(\ell) \in \mathcal{N}_{\epsilon \ell}(A)$, the middle third $\mathfrak{g}\left(\left[\frac{\ell}{3}, \frac{2 \ell}{3}\right]\right)$ is contained in $\mathcal{N}_{M}(A)$;

(Qconv) (uniform quasi-convexity of pieces) there exists $t>0$ and $K_{0} \geq 0$ such that for every $A \in \mathcal{A}, K \geq K_{0}$ and $x, y \in \mathcal{N}_{K}(A)$, every geodesic joining $x$ and $y$ in $X$ is contained in $\mathcal{N}_{t K}(A)$.

Proof. Assume that $X$ is ATG with respect to $\mathcal{A}$. We prove that $(X, \mathcal{A})$ satisfies properties $\left(\beta_{2}\right)$ and (Qconv). Property $\left(\beta_{3}\right)$ is obviously satisfied, as it is a particular case of property $\left(\alpha_{3}\right)$.

The uniform quasi-convexity condition (Qconv) is satisfied by Lemma 4.3 in [DS05b].

Property $\left(\beta_{2}\right)$ can be obtained for any $\epsilon<\frac{1}{6 t}$, where $t$ is the constant from (Qconv), as follows. Consider a geodesic $g$ of length $\ell$ and $A \in \mathcal{A}$ as in $\left(\beta_{2}\right)$. We may assume that $\epsilon \ell \geq K_{0}$, otherwise $g$ would be contained in $\mathcal{N}_{\frac{K_{0}}{2 \epsilon}}$. By (Qconv) the geodesic $\mathrm{g}$ is then contained in $\mathcal{N}_{t \in \ell}(A)$. If $\theta=t \epsilon<\frac{1}{6}$ then by Theorem 4.9 and Remark 4.10 there exists $M=M(\theta)$ such that $\mathfrak{g}\left(\left[0, \frac{\ell}{3}\right]\right)$ and $\mathfrak{g}\left(\left[\frac{2 \ell}{3}, \ell\right]\right)$ intersect $\mathcal{N}_{M}(A)$. Uniform convexity implies that $\mathrm{g}\left(\left[\frac{\ell}{3}, \frac{2 \ell}{3}\right]\right)$ is contained in $\mathcal{N}_{t M^{\prime}}(A)$, where $M^{\prime}=\max \left(M, D_{0}\right)$.

It remains to prove the converse statements in Theorem 4.22, i.e., that any of the triples of properties $\left(\alpha_{1}\right) \&\left(\alpha_{2}\right) \&\left(\beta_{3}\right),\left(\alpha_{1}\right) \&\left(\beta_{2}\right) \&\left(\beta_{3}\right)$ or $\left(\alpha_{1}\right) \&(\mathrm{Qconv}) \&\left(\beta_{3}\right)$ implies that $X$ is ATG with respect to $\mathcal{A}$. We begin by proving that the first two triples of properties are equivalent to the last one.

The implication $\left(\alpha_{1}\right) \&\left(\alpha_{2}\right) \Rightarrow$ (Qconv) is proved in [DS05b, Lemma 4.3].

$\left(\alpha_{1}\right) \&\left(\beta_{2}\right) \Rightarrow(\mathrm{Q}$ conv $)$. The constant $K_{0}$ in (Qconv) is taken equal to the constant $M$ in $\left(\beta_{2}\right)$.

Suppose by contradiction that for every $n \in \mathbb{N}^{*}$ there exists $A_{n} \in \mathcal{A}, K_{n} \geq M$ and $x_{n}, y_{n} \in \mathcal{N}_{K_{n}}\left(A_{n}\right)$ such that a geodesic $\left[x_{n}, y_{n}\right]$ is not contained in $\mathcal{N}_{n K_{n}}\left(A_{n}\right)$. For each $n \in \mathbb{N}^{*}$ we define $D_{n}$ to be the infimum over the $\operatorname{distances} \operatorname{dist}\left(x_{n}, y_{n}\right)$ 
between pairs of points satisfying the properties above for some set in $\mathcal{A}$. In what follows we assume that we chose $x_{n}, y_{n}$ at distance $\delta_{n} \leq D_{n}+1$ of each other. Since $\left[x_{n}, y_{n}\right]$ is in $\mathcal{N}_{\delta_{n} / 2}\left(\left\{x_{n}, y_{n}\right\}\right) \subset \mathcal{N}_{\delta_{n} / 2+K_{n}}\left(A_{n}\right)$ it follows that $\frac{1}{2 n-2} \delta_{n} \geq K_{n}$. In particular for $n$ large enough $K_{n}<\epsilon \delta_{n}$, where $\epsilon>0$ is the constant in $\left(\beta_{2}\right)$. It follows that the middle third $\left[a_{n}, b_{n}\right]$ of $\left[x_{n}, y_{n}\right]$ is contained in $\mathcal{N}_{M}\left(A_{n}\right)$. Since $K_{n} \geq M$, the fact that $\left[x_{n}, y_{n}\right] \not \subset \mathcal{N}_{n K_{n}}\left(A_{n}\right)$ implies that either $\left[x_{n}, a_{n}\right]$ or $\left[b_{n}, y_{n}\right]$ is not contained in $\mathcal{N}_{n K_{n}}\left(A_{n}\right)$. It follows that $D_{n} \leq \frac{\delta_{n}}{3} \leq \frac{D_{n}+1}{3}$, hence that the sequence $\left(D_{n}\right)$ is uniformly bounded. This contradicts the fact that $D_{n} \geq(2 n-2) M$.

$\left(\alpha_{1}\right) \&($ Qconv $) \&\left(\beta_{3}\right) \Rightarrow\left(\alpha_{2}\right),\left(\beta_{2}\right)$. Let $\mathrm{g}:[0, \ell] \rightarrow X$ be a geodesic with endpoints $x=\mathfrak{g}(0)$ and $y=\mathfrak{g}(\ell)$ contained in $\mathcal{N}_{\varepsilon \ell}(A)$ for some $A \in \mathcal{A}$. We shall prove that for a fixed positive constant $D$, the geodesic $\mathrm{g}$ intersects $\mathcal{N}_{D}(A)$.

According to (Qconv), the geodesic $\mathrm{g}$ is contained in $\mathcal{N}_{t \varepsilon \ell}(A)$.

Notation 4.23. We denote $t \varepsilon$ by $\epsilon$ and we assume in what follows that $\epsilon<\frac{1}{8}$. We denote by $D$ the maximum between $t K_{0}+4 v \theta, \chi$ and $\operatorname{diam}_{\delta}$ (with the notation 4.11) for $\delta=\max \left(\chi, t K_{0}\right)$. Here $t$ and $K_{0}$ are the constants appearing in (Qconv), while $\nu, \theta, \chi$ are the constants appearing in $\left(\beta_{3}\right)$.

Suppose by contradiction that $\mathrm{g}$ does not intersect $\mathcal{N}_{D}(A)$. Note that since $\epsilon \ell \geq D$ it follows that $\ell>8 D$.

Consider $x^{\prime}$ and $y^{\prime}$ points in $A$ such that $\operatorname{dist}\left(x, x^{\prime}\right)$ and $\operatorname{dist}\left(y, y^{\prime}\right)$ are at most $\varepsilon \ell$. By (Qconv), a geodesic $\mathfrak{g}^{\prime}$ joining $x^{\prime}$ and $y^{\prime}$ is contained in $\mathcal{N}_{t K_{0}}(A)$.

Let $c \in \mathfrak{g}$ and $c^{\prime} \in \mathfrak{g}^{\prime}$ be two points such that $\operatorname{dist}\left(c, c^{\prime}\right)=\operatorname{dist}\left(\mathfrak{g}, \mathfrak{g}^{\prime}\right)$. Without loss of generality we may suppose that $\operatorname{dist}(x, c) \geq \frac{\ell}{2}$. We may also suppose that $\operatorname{dist}\left(x, x^{\prime}\right)=\operatorname{dist}\left(x, \mathrm{~g}^{\prime}\right)$. In order to transform the 4-gon of vertices $x, x^{\prime}, c, c^{\prime}$, into a fat polygon we make the following choices. Let $x_{1}$ be the point on $g$ between $x$ and $c$ which is farthest from $x$ and at distance at most $2 v \theta$ from $\left[x, x^{\prime}\right]$. Let $x_{2}$ be the farthest from $x$ point on $\left[x, x^{\prime}\right]$ which is at distance $2 v \theta$ from $x_{1}$.

We prove in the sequel that the geodesic pentagon of vertices $x_{1}, x_{2}, x^{\prime}, c^{\prime}, c$ is $(\theta, 2 v)$-fat. To simplify we shall denote its edges by $[v, w]$ if $v, w$ are two consecutive vertices, keeping in mind that $\left[x_{1}, c\right] \subset \mathfrak{g}$ and that $\left[x^{\prime}, c^{\prime}\right] \subset \mathfrak{g}^{\prime}$.

$\left(\right.$ Fat $\left._{1}\right)$ : A point in $\left[c, c^{\prime}\right] \backslash \mathcal{N}_{2 \theta}\left(\left\{c, c^{\prime}\right\}\right)$ is at distance at least $\left(\frac{1}{2}-2 \epsilon\right) \ell$ from $\left[x, x^{\prime}\right]$, hence at distance at least $\left(\frac{1}{2}-2 \epsilon\right) \ell-2 v \theta$ of $\left[x_{1}, x_{2}\right]$. Since $\ell>8 D>32 v \theta$, it follows that $\left[c, c^{\prime}\right] \backslash \mathcal{N}_{2 \theta}\left(\left\{c, c^{\prime}\right\}\right)$ is at distance at least $\theta$ from $\left[x_{1}, x_{2}\right] \cup\left[x_{2}, x^{\prime}\right]$.

The choice of $c, c^{\prime}$ implies that all points in $\left[c, c^{\prime}\right] \backslash \mathcal{N}_{2 \theta}\left(\left\{c, c^{\prime}\right\}\right)$ are at distance at least $2 \theta$ from $g$ and from $\mathfrak{g}^{\prime}$.

The points in $\left[x_{1}, c\right] \backslash \mathcal{N}_{2 \theta}\left(\left\{x_{1}, c\right\}\right)$ are at distance at least $D-t K_{0}$ from $\mathrm{g}^{\prime}$, and at distance at least $2 v \theta$ from $\left[x_{2}, x^{\prime}\right]$. Lemma 4.7 allows to conclude that $\left[x_{1}, c\right]$ satisfies property $\left(\mathrm{Fat}_{1}\right)$.

The distance between $\left[x_{1}, x_{2}\right]$ and $\left[c, c^{\prime}\right]$ is at least $\left(\frac{1}{2}-2 \epsilon\right) \ell-2 \nu \theta$, and the one between $\left[x_{1}, x_{2}\right]$ and $\left[x^{\prime}, c^{\prime}\right]$ is at least $D-t K_{0}-2 v \theta$. Thus, it suffices to verify 
that the distance between $\left[x_{1}, x_{2}\right] \backslash \mathcal{N}_{2 \theta}\left(\left\{x_{1}, x_{2}\right\}\right)$ and $\left[c, x_{1}\right] \cup\left[x_{2}, x^{\prime}\right]$ is at least $\theta$. According to the choices of $x_{1}, x_{2}$ this distance is $2 \theta$. This, and Lemma 4.7 also imply that $\left[x_{2}, x^{\prime}\right]$ satisfies $\left(\right.$ Fat $\left._{1}\right)$.

The fact that the edge $\left[x^{\prime}, c^{\prime}\right]$ is at distance at least $D-t K_{0}-2 v \theta$ from $\mathrm{g} \cup\left[x_{1}, x_{2}\right]$, together with Lemma 4.7, imply that $\left[x^{\prime}, c^{\prime}\right]$ satisfies $\left(\mathrm{Fat}_{1}\right)$.

$\left(\right.$ Fat $\left._{2}\right)$ : The vertex $c^{\prime}$ is at distance at least $D-t K_{0}-2 v \theta$ from $\left[c, x_{1}\right] \cup\left[x_{1}, x_{2}\right]$ and at distance at least $\left(\frac{1}{2}-2 \epsilon\right) \ell$ from $\left[x_{2}, x^{\prime}\right]$.

The vertex $c$ is at distance at least $D-t K_{0}$ from $\left[x^{\prime}, c^{\prime}\right]$ and at distance at least $\left(\frac{1}{2}-2 \epsilon\right) \ell-2 v \theta$ from $\left[x_{1}, x_{2}\right] \cup\left[x_{2}, x^{\prime}\right]$.

We have chosen $x_{1}$ at distance $2 v \theta$ from $\left[x_{2}, x^{\prime}\right]$. The same vertex is at distance at least $D-t K_{0}$ from $\left[x^{\prime}, c^{\prime}\right]$, and at least $\left(\frac{1}{2}-2 \epsilon\right) \ell-2 v \theta$ from $\left[c, c^{\prime}\right]$.

Similarly, $x_{2}$ is at distance $2 \nu \theta$ from $\left[x_{1}, c\right]$, at distance at least $\left(\frac{1}{2}-2 \epsilon\right) \ell-2 \nu \theta$ from $\left[c, c^{\prime}\right]$ and at least $D-t K_{0}-2 v \theta$ from $\left[x^{\prime}, c^{\prime}\right]$.

The vertex $x^{\prime}$ is at distance at least $D-t K_{0}-2 v \theta$ from $\left[c, x_{1}\right] \cup\left[x_{1}, x_{2}\right]$ and at least $\left(\frac{1}{2}-2 \epsilon\right) \ell$ from $\left[c, c^{\prime}\right]$.

The pentagon of vertices $x_{1}, x_{2}, x^{\prime}, c^{\prime}, c$ is $(\theta, 2 v)$-fat. Lemma 4.8 and the fact that $\operatorname{dist}\left(c, c^{\prime}\right)=\operatorname{dist}\left(c,\left[c^{\prime}, x^{\prime}\right]\right)$ implies that by adding a vertex on $\left[c, c^{\prime}\right]$ this pentagon becomes a hexagon $(\theta, v)$-fat. Therefore by $\left(\beta_{3}\right)$ it is contained in $\mathcal{N}_{\chi}\left(A^{\prime}\right)$ for some $A^{\prime} \in \mathcal{A}$. In particular the edge $\left[x^{\prime}, c^{\prime}\right]$ is contained in $\mathcal{N}_{\chi}\left(A^{\prime}\right) \cap \mathcal{N}_{t K_{0}}(A)$. This edge has length at least $\frac{\ell}{4}>2 D$ and $D$ is at least $\operatorname{diam}_{\delta}$ for $\delta=\max \left(\chi, t K_{0}\right)$. It follows that $A=A^{\prime}$ and that $D<\chi$, which is a contradiction.

We conclude that property $\left(\alpha_{2}\right)$ is satisfied for $\varepsilon<\frac{1}{8 t}$ and for $D$ chosen above.

Property $\left(\beta_{2}\right)$ is obtained as follows. If a geodesic $\mathrm{g}:[0, \ell] \rightarrow X$ joins two points in $\mathcal{N}_{\delta \ell}(A)$ then it is contained in $\mathcal{N}_{t \delta \ell}(A)$ by (Qconv). If $\delta<\frac{\varepsilon}{3 t}$ then by $\left(\alpha_{2}\right)$ the subgeodesics $g\left(\left[0, \frac{\ell}{3}\right]\right)$ and $g\left(\left[\frac{2 \ell}{3}, \ell\right]\right)$ intersect $\mathcal{N}_{M}(A)$. Then by (Qconv), $g\left(\left[\frac{\ell}{3}, \frac{2 \ell}{3}\right]\right)$ is contained in $\mathcal{N}_{t M}(A)$.

In order to finish the proof of Theorem 4.22 it now suffices to prove the following. $\left(\alpha_{1}\right) \&\left(\alpha_{2}\right) \&(\mathrm{Qconv}) \&\left(\beta_{3}\right) \Rightarrow X$ is ATG with respect to $\mathcal{A}$ in the sense of Definition 4.1 .

In an asymptotic cone $\operatorname{Con}_{\omega}(X ; e, d)$, the limit sets in $\mathcal{A}_{\omega}$ are closed. Property (Qconv) easily implies that all subsets in $\mathcal{A}_{\omega}$ are geodesic.

The fact that two distinct limit subsets from $\mathcal{A}_{\omega}$ intersect in at most a point (i.e., property $\left.\left(T_{1}\right)\right)$ is deduced from $\left(\alpha_{1}\right)$ and $\left(\alpha_{2}\right)$ as in [DS05b, Lemma 4.5]. Properties $\left(T_{1}\right) \&\left(\beta_{3}\right)$ imply that non-trivial simple geodesic triangles are contained in subsets from $\mathcal{A}_{\omega}$ (i.e. property $\left(T_{2}\right)$ ) by Corollary 4.20 .

In the new definitions of ATG metric spaces provided by Theorem 4.22 property $\left(\beta_{3}\right)$ can be still weakened, in the following sense.

Proposition 4.24. For any $\eta>0$ property $\left(\beta_{3}\right)$ can be replaced in Theorem 4.22 by the following version of it, in which only large hexagons are taken into account: 
$\left(\beta_{3}^{\eta}\right)$ there exists $\theta>0, v \geq 8$ and $\chi>0$ such that any geodesic hexagon $(\theta, v)$-fat of diameter at least $\eta$ is contained in $\mathcal{N}_{\chi}(A)$, for some $A \in \mathcal{A}$.

Proof. Indeed, as a sufficient condition $\left(\beta_{3}\right)$ is used in combination with Proposition 4.14 to prove that in any asymptotic cone property $\left(\Pi_{3}\right)$ holds (i.e., every simple non-trivial triangle with edges limit geodesics is contained in a $\operatorname{limit}_{\text {set }} \lim _{\omega}\left(A_{n}\right)$ with $A_{n} \in \mathcal{A}$ ).

Given, in an asymptotic cone, such a simple non-trivial triangle with edges limit geodesics, and the sequence of hexagons $H_{n}$ provided for this triangle by Proposition 4.14 , the sequence of diameters of $H_{n}$ has $\omega$-limit $\infty$. Property $\left(\beta_{3}^{\eta}\right)$ suffices therefore to obtain property $\left(\Pi_{3}\right)$.

Property $\left(\beta_{3}\right)$ is also used in the proof of Theorem 4.22, combined with $\left(\alpha_{1}\right)$ and (Qconv), to deduce property $\left(\alpha_{2}\right)$. There also it can be replaced by $\left(\beta_{3}^{\eta}\right)$, for any large $\eta$. Indeed, it suffices to take, in the proof of the implication $\left(\alpha_{1}\right) \&(\mathrm{Qconv}) \&\left(\beta_{3}\right) \Rightarrow$ $\left(\alpha_{2}\right)$, the constant $D$ larger than $t K_{0}+\eta$ to obtain that the geodesic pentagon with vertices $x_{1}, x_{2}, x^{\prime}, c^{\prime}, c$ has diameter at least $\operatorname{dist}\left(c, c^{\prime}\right) \geq D-t K_{0}>\eta$. That pentagon is $(\theta, 2 v)$-fat, hence by Lemma 4.8 it can be made into a hexagon $(\theta, v)$ fat of diameter larger than $\eta$; therefore by $\left(\beta_{3}^{\eta}\right)$ it is contained in $\mathcal{N}_{\chi}\left(A^{\prime}\right)$ for some $A^{\prime} \in \mathcal{A}$. The rest of the argument is carried out similarly.

Corollary 4.25. Let $\mathcal{A}_{\text {red }}^{\eta}$ be the set of $A \in \mathcal{A}$ such that $\mathcal{N}_{\chi}(A)$ contains a $(\theta, v)$-fat geodesic hexagon of diameter at least $\eta$. Then the space $X$ is ATG with respect to $\mathcal{A}_{\text {red }}^{\eta}$.

Proof. Since $\mathcal{A}_{\text {red }}^{\eta} \subset \mathcal{A}$, and $\mathcal{A}$ satisfies $\left(\alpha_{1}\right)$ and $\left(\beta_{2}\right)$, the same properties are satisfied by $\mathcal{A}_{\text {red }}^{\eta}$. Property $\left(\beta_{3}^{\eta}\right)$ is also satisfied by $\mathcal{A}_{\text {red }}^{\eta}$, hence by Proposition 4.24, $X$ is ATG with respect to $\mathcal{A}_{\text {red }}^{\eta}$.

Corollary 4.26. For every $\lambda>0$ the space $X$ is also ATG with respect to the subset $\mathcal{A}_{\lambda}$ in $\mathcal{A}_{\text {composed }}$ of all the subsets of diameter at least $\lambda$ in $\mathcal{A}$.

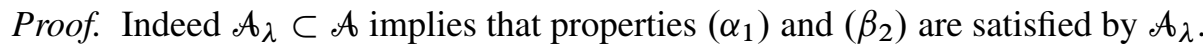

Let $\eta=\lambda+2 \chi$. Then $\mathcal{A}_{\text {red }}^{\eta} \subset \mathcal{A}_{\lambda}$, which implies that property $\left(\beta_{3}^{\eta}\right)$ is satisfied by $\mathcal{A}_{\lambda}$. By Proposition 4.24, $X$ is ATG with respect to $\mathcal{A}_{\lambda}$.

4.4. New definition, closer to the definition of hyperbolicity. In [DS05a] a version for groups of the following notion has been introduced.

Definition 4.27. Let $X$ be a geodesic metric space and let $\mathcal{A}$ be a collection of subsets of $X$. We say that $X$ is $(*)$-asymptotically tree-graded with respect to $\mathcal{A}$ if for every $C \geq 0$ there exist two constants $\sigma$ and $\delta$ such that every triangle $x y z$ with $(1, C)$-almost geodesic edges is in one of the following two cases: 
(C) there exists $a \in X$ such that $\bar{B}(a, \sigma)$ intersects each of the sides of the triangle;

(P) there exists $A \in \mathcal{A}$ such that $\overline{\mathcal{N}}_{\sigma}(A)$ intersects each of the sides of the triangle, and the entrance (resp. exit) points $x_{1}, y_{1}, z_{1}$ (resp. $y_{2}, z_{2}, x_{2}$ ) of the sides $[x, y],[y, z],[z, x]$ in (from) $\overline{\mathcal{N}}_{\sigma}(A)$ satisfy

$$
\operatorname{dist}\left(x_{1}, x_{2}\right)<\delta, \quad \operatorname{dist}\left(y_{1}, y_{2}\right)<\delta, \quad \operatorname{dist}\left(z_{1}, z_{2}\right)<\delta .
$$

See Figure 5, taken from [DS05b].

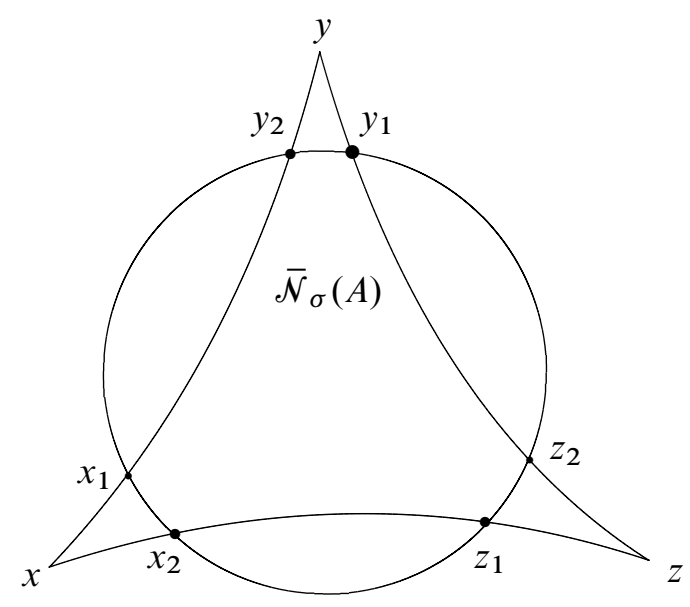

Figure 5. Case (P) of Definition 4.27.

In Definition 4.27, (C) stands for "center", since the point $a$ is in some sense a center of the triangle $x y z$; $(\mathrm{P})$ stands for "piece", since in this case the triangle $x y z$ has a central piece.

Remark 4.28. If $X$ is a geodesic metric space in which for some constant $\sigma>0$ every geodesic triangle satisfies property $(\mathrm{C})$, then $X$ is a hyperbolic space. Conversely, in a hyperbolic geodesic metric space for every $L \geq 1$ and $C \geq 0$ there exists $\sigma>0$ such that every triangle with $(L, C)$-quasi-geodesic edges satisfies property $(C)$.

Remarks 4.29. (1) If a metric space $X$ is ATG with respect to a collection of subsets $\mathcal{A}$ then $X$ is $(*)$-ATG with respect to $\mathcal{A}$; this follows from [DS05b, Corollary 8.14 and Lemma 8.19].

Moreover, according to [DS05b, Corollary 8.14] if a geodesic triangle is in case (P) then for every $\sigma^{\prime} \geq \sigma$ there exists $\delta^{\prime}$ such that the pairs of entrance points in $\overline{\mathcal{N}}_{\sigma^{\prime}}(A)$ are at distance at most $\delta^{\prime}$. 
(2) The notion of $(*)$-ATG space is weaker than the one of ATG space. For instance if $X$ is a geodesic hyperbolic space and if $\mathcal{A}$ is any collection of subsets covering $X$, then $X$ is $(*)$-ATG with respect to $\mathcal{A}$, and the collection $\mathcal{A}$ needs not satisfy property $\left(\alpha_{1}\right)$ requiring uniformly bounded diameter for intersections of bounded radius tubular neighborhoods of distinct subsets in $\mathcal{A}$, or property (Qconv) requiring uniform quasi-convexity for subsets in $\mathcal{A}$.

It turns out nevertheless that one can formulate an equivalent definition of ATG metric spaces using the $(*)$-property.

Theorem 4.30. Let ( $X$, dist) be a geodesic metric space and let $\mathcal{A}$ be a collection of subsets of $X$. The metric space $X$ is asymptotically tree-graded with respect to $\mathcal{A}$ if and only if $(X, \mathcal{A})$ satisfy properties $\left(\alpha_{1}\right)$ and $\left(\alpha_{2}\right)$ from Theorem 4.9 , and moreover $X$ is $(*)-A T G$ with respect to $\mathcal{A}$.

Proof. The direct implication has already been discussed, we now prove the converse statement, that is, we prove that $\left(\alpha_{1}\right) \&\left(\alpha_{2}\right) \&(*)$ imply that $X$ is asymptotically treegraded with respect to $\mathcal{A}$.

In order to simplify some technical arguments, we make the assumption that for all $C>0$ the constant $\sigma$ in the (*)-property is larger than the constant $M$ appearing in property $\left(\alpha_{2}\right)$. By Remark 4.29, (1), if $X$ is ATG then such a choice of $\sigma$ is possible.

By [DS05b, Lemma 4.3] properties $\left(\alpha_{1}\right) \&\left(\alpha_{2}\right)$ imply property (Qconv) on the quasi-convexity of subsets in $\mathcal{A}$. From this property follows that in any asymptotic cone $\operatorname{Con}_{\omega}(X ; e, d)$ the collection $\mathcal{A}_{\omega}$ is composed of closed geodesic subsets.

Again $\left(\alpha_{1}\right) \&\left(\alpha_{2}\right)$ imply property $\left(T_{1}\right)$ for $\mathcal{A}_{\omega}$ (i.e. that distinct subsets from $\mathcal{A}_{\omega}$ intersect in at most one point). According to Corollary 4.19, in order to conclude that $\operatorname{Con}_{\omega}(X ; e, d)$ is tree-graded with set of pieces $\mathcal{A}_{\omega}$ (and thus finish the proof of Theorem 4.30), it suffices to prove property $\left(\Pi_{3}\right)$, i.e., that simple non-trivial triangles with edges limit geodesics are contained in subsets from $\mathcal{A}_{\omega}$.

We split the proof of $\left(\Pi_{3}\right)$ into several steps, which we formulate as separate lemmata. The main and most difficult step is Lemma 4.33 stating that $\left(\alpha_{1}\right) \&\left(\alpha_{2}\right) \&(*)$ imply that fat quadrilaterals stay close to subsets in $\mathcal{A}$. From Lemma 4.33 it may be first deduced that property $\left(\Pi_{2}\right)$ is satisfied by $\mathcal{A}_{\omega}$ in any asymptotic cone (Lemma 4.35), then that property $\left(\Pi_{3}\right)$ is satisfied by $\mathscr{A}_{\omega}$ (Lemma 4.36).

Lemma 4.31 (entrance points in nested tubular neighborhoods). Let (X, dist) be a geodesic metric space and let $\mathcal{A}$ be a collection of subsets of $X$ satisfying property $\left(\alpha_{2}\right)$ for some $\varepsilon \in[0,1 / 2)$ and $M>0$.

Let $\mu \geq v \geq M$, let $\mathrm{g}$ be a geodesic and $A$ a subset in $\mathcal{A}$ such that $\mathrm{g}$ intersects $\overline{\mathcal{N}}_{\nu}(A)$. If $e_{\mu}$ and $e_{\nu}$ are the entrance points of $\mathrm{g}$ in $\overline{\mathcal{N}}_{\mu}(A)$ and respectively $\overline{\mathcal{N}}_{\nu}(A)$ then $\operatorname{dist}\left(e_{\mu}, e_{\nu}\right) \leq \frac{\mu}{\varepsilon}$. 
Proof. If $\varepsilon \operatorname{dist}\left(e_{\mu}, e_{\nu}\right)>\mu$ then by $\left(\alpha_{2}\right)$ the sub-arc of $g$ between $e_{\mu}$ and $e_{\nu}$ intersects $\mathcal{N}_{M}(A) \subset \mathcal{N}_{v}(A)$, which contradicts the definition of $e_{\nu}$.

Lemma 4.32. If $(X, \mathcal{A})$ satisfy properties $(*)$ and $\left(\alpha_{2}\right)$ then for every $C \geq 0$ there exist $\kappa \geq 0$ and $\lambda \geq 0$ such that for any two geodesics $\mathrm{g}$ and $\mathrm{g}^{\prime}$ in $X$ with $\mathrm{g}_{-}=\mathrm{g}_{-}^{\prime}$ and $\operatorname{dist}\left(\mathfrak{g}_{+}, \mathfrak{g}_{+}^{\prime}\right) \leq C$ the following holds:

(1) any point $z$ on $\mathrm{g}^{\prime}$ is either contained in $\overline{\mathcal{N}}_{\kappa}(\mathrm{g})$ or it is contained in $\overline{\mathcal{N}}_{\kappa}(A)$ for some $A \in \mathcal{A}$ such that $\overline{\mathcal{N}}_{\kappa}(A)$ intersects $\mathrm{g}$;

(2) if $\overline{\mathcal{N}}_{\kappa}(A)$ intersects $\mathrm{g}$ and $\mathrm{g}^{\prime}$, and $e, f$ and $e^{\prime}, f^{\prime}$ are the entrance and exit points from $\overline{\mathcal{N}}_{\kappa}(A)$ of $\mathrm{g}$ and respectively $\mathrm{g}^{\prime}$, then $\operatorname{dist}\left(e, e^{\prime}\right), \operatorname{dist}\left(f, f^{\prime}\right) \leq \lambda$.

Proof. Let $\mathfrak{p}$ be the path $\mathfrak{g} \sqcup\left[\mathfrak{g}_{+}, \mathfrak{g}_{+}^{\prime}\right]$, where $\left[\mathfrak{g}_{+}, \mathfrak{g}_{+}^{\prime}\right]$ is a geodesic segment joining $\mathfrak{g}_{+}$and $\mathfrak{g}_{+}^{\prime}$. It is a $(1,2 C)$-almost geodesic. Let $\sigma$ and $\delta$ be the constants of property $(*)$ for $2 C$, and let $z$ be an arbitrary point on $\mathfrak{g}^{\prime}$, dividing $\mathfrak{g}^{\prime}$ into two sub-arcs, $\mathfrak{g}_{1}$ and $\mathfrak{g}_{2}$. The triangle $\Delta$ of edges $\mathfrak{g}_{1}, \mathfrak{g}_{2}$ and $\mathfrak{p}$ is either in case $(\mathrm{C})$ or in case (P) of Definition 4.27.

If it is in case (C) then there exist $a_{1} \in \mathfrak{g}_{1}, a_{2} \in \mathfrak{g}_{2}$ and $b \in \mathfrak{p}$ such that the set $\left\{a_{1}, a_{2}, b\right\}$ has diameter at most $2 \sigma$. The point $z$ is on a geodesic joining $a_{1}$ and $a_{2}$, hence it is at distance at most $3 \sigma$ from $b$, thus it is contained in $\overline{\mathcal{N}}_{3 \sigma+C}(\mathrm{~g})$.

If $\Delta$ is in case (P) then there exists $A \in \mathcal{A}$ with $\overline{\mathcal{N}}_{\sigma}(A)$ intersecting $\mathfrak{g}_{1}, \mathfrak{g}_{2}$ and $\mathfrak{p}$. Let $x_{1}, z_{1}, z_{2}, y_{1}$ and $x_{2}, y_{2}$ be the entrance and exit points from $\overline{\mathcal{N}}_{\sigma}(A)$ of $\mathfrak{g}_{1}, \mathfrak{g}_{2}$ and $p$ respectively. Then $\operatorname{dist}\left(x_{1}, x_{2}\right)$, dist $\left(y_{1}, y_{2}\right)$ and $\operatorname{dist}\left(z_{1}, z_{2}\right)$ are all at most $\delta$. Since $z$ is on a geodesic joining $z_{1}$ and $z_{2}, z \in \overline{\mathcal{N}}_{\sigma+\delta / 2}(A)$. Note that $\overline{\mathcal{N}}_{\sigma}(A)$ intersects $\mathfrak{p}$, therefore $\overline{\mathcal{N}}_{\sigma+C}(A)$ intersects $\mathrm{g}$.

Take $\kappa=\max \left(3 \sigma+C, \sigma+\frac{\delta}{2}, \sigma+C\right)$.

The points $x_{1}$ and $y_{1}$ are the entrance and respectively the exit point of $\mathrm{g}^{\prime}$ from $\overline{\mathcal{N}}_{\sigma}(A)$. If we consider $e^{\prime}$ and $f^{\prime}$ the entrance and exit points of $\mathrm{g}^{\prime}$ from $\overline{\mathcal{N}}_{\kappa}(A)$, Lemma 4.31 implies that $\operatorname{dist}\left(x_{1}, e^{\prime}\right)$ and $\operatorname{dist}\left(y_{1}, f^{\prime}\right)$ are at most $\frac{\kappa}{\varepsilon}$. Hence $\operatorname{dist}\left(e^{\prime}, x_{2}\right)$ and $\operatorname{dist}\left(f^{\prime}, y_{2}\right)$ are at most $\frac{\kappa}{\varepsilon}+\delta$.

Let $e$ and $f$ be the entrance and exit points of $g$ into (from) $\overline{\mathcal{N}}_{\kappa}(A)$. If either $x_{2}$ or both $x_{2}$ and $y_{2}$ are in $g$ then they are the entrance and respectively the exit point of $\mathrm{g}$ from $\overline{\mathcal{N}}_{\sigma}(A)$. Lemma 4.31 implies that either $\operatorname{dist}\left(x_{2}, e\right)$ or both $\operatorname{dist}\left(x_{2}, e\right)$ and $\operatorname{dist}\left(y_{2}, f\right)$ are at most $\frac{\kappa}{\varepsilon}$, hence that either $\operatorname{dist}\left(e, e^{\prime}\right)$ or both $\operatorname{dist}\left(e, e^{\prime}\right), \operatorname{dist}\left(f, f^{\prime}\right)$ are $O(1)$.

Assume that $y_{2} \in\left[\mathfrak{g}_{+}, \mathfrak{g}_{+}^{\prime}\right]$. Then $\mathfrak{g}_{+}$is in $\overline{\mathcal{N}}_{\kappa}(A)$, hence $\mathfrak{g}_{+}=f$. It follows that $\operatorname{dist}\left(f, y_{2}\right) \leq C$ and that $\operatorname{dist}\left(f, f^{\prime}\right) \leq C+\frac{\kappa}{\varepsilon}+\delta$.

Assume that $x_{2} \in\left[\mathfrak{g}_{+}, \mathfrak{g}_{+}^{\prime}\right]$. The point $\mathfrak{g}_{+}$is in $\overline{\mathcal{N}}_{\kappa}(A)$, and if $\varepsilon \operatorname{dist}\left(e, \mathfrak{g}_{+}\right)>\kappa$ then $\mathrm{g}$ intersects $\mathcal{N}_{M}(A)$ between $e$ and $\mathfrak{g}_{+}$.

In the beginning of the proof of Theorem 4.30 we made the assumption that for all $C>0$ the constant $\sigma$ in the (*)-property is larger than the constant $M$ in property 
$\left(\alpha_{2}\right)$. It follows that $\mathcal{N}_{M}(A) \subset \mathcal{N}_{\sigma}(A)$, hence $g$ intersects $\mathcal{N}_{\sigma}(A)$ between $e$ and $\mathfrak{g}_{+}$. This contradicts the fact that $x_{2}$ is the entrance point of $\mathfrak{p}$ into $\overline{\mathcal{N}}_{\sigma}(A)$. Thus $\operatorname{dist}\left(e, \mathfrak{g}_{+}\right) \leq \frac{\kappa}{\varepsilon}$ and $\operatorname{dist}\left(e, x_{2}\right) \leq \frac{\kappa}{\varepsilon}+C$, whence $\operatorname{dist}\left(e, e^{\prime}\right) \leq 2 \frac{\kappa}{\varepsilon}+C+\delta$.

Lemma 4.33. Assume that ( $X$, dist) is $(*)-A T G$ with respect to $\mathcal{A}$, and that $(X, \mathcal{A})$ satisfies properties $\left(\alpha_{1}\right),\left(\alpha_{2}\right)$ from Theorem 4.9 and the uniform quasi-convexity condition (Qconv).

Then there exist $\theta>0, v \geq 8$ and $\chi>0$ such that any geodesic quadrilateral which is $(\theta, v)$-fat is contained in $\mathcal{N}_{\chi}(A)$ for some $A \in \mathcal{A}$.

Proof. Let $P$ be a $(\theta, v)$-fat geodesic quadrilateral with vertices $x, y, z, w$ in counterclockwise order. Let $[x, z]$ be a geodesic joining the opposite vertices $x$ and $z$.

Case 1. Assume that both geodesic triangles $x y z$ and $x z w$ have a center, that is, they are in case (C) of Definition 4.27. Then there exists $a_{1} \in[x, y], a_{2} \in[y, z]$ and $a_{3} \in[x, z]$ such that the set $\left\{a_{1}, a_{2}, a_{2}\right\}$ has diameter at most $2 \sigma$. Likewise there exists $b_{1} \in[z, w], b_{2} \in[w, x]$ and $b_{3} \in[z, x]$ such that $\left\{b_{1}, b_{2}, b_{3}\right\}$ has diameter at most $2 \sigma$. If $\theta>2 \sigma$ then $a_{1}, a_{2} \in B(y, 2 \theta)$ and $b_{1}, b_{2} \in B(w, 2 \theta)$.

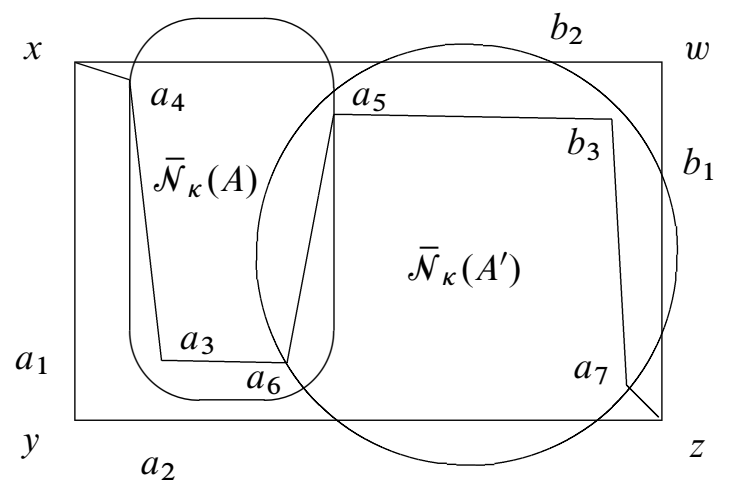

Figure 6. Case 1 in the proof of Lemma 4.33.

Without loss of generality we may assume that $a_{3} \in\left[x, b_{3}\right]$.

Notation 4.34. For $C=\max (2 \sigma, \delta)$ we denote by $\kappa$ and $\lambda$ the constants given by Lemma 4.32.

Lemma 4.32 applied to $\left[x, b_{2}\right]$ and to $a_{3} \in\left[x, b_{3}\right]$ implies that either $a_{3} \in$ $\overline{\mathcal{N}}_{\kappa}\left(\left[x, b_{2}\right]\right)$, or $a_{3} \in \overline{\mathcal{N}}_{\kappa}(A)$ such that the entrance respectively exit point, $a_{4}, a_{5}$, of $\left[x, b_{3}\right]$ into (from) $\overline{\mathcal{N}}_{\kappa}(A)$ are at distance at most $\lambda$ from $\left[x, b_{2}\right]$. If $\theta>2 \sigma+\kappa$ then by Lemma 4.6 the first case cannot occur. 
In the second case we have that dist $\left(a_{3},\left\{a_{4}, a_{5}\right\}\right)$ is at least $\theta-2 \sigma-\lambda$. Lemma 4.32 applied to $\left[z, a_{2}\right]$ and to $a_{5} \in\left[z, a_{3}\right]$ implies that either $a_{5} \in \overline{\mathcal{N}}_{\kappa}\left(\left[z, a_{2}\right]\right)$ or that $a_{5} \in$ $\overline{\mathcal{N}}_{\kappa}\left(A^{\prime}\right)$ such that the entrance and the exit point $a_{6}, a_{7}$, of $\left[a_{3}, z\right]$ from $\overline{\mathcal{N}}_{\kappa}\left(A^{\prime}\right)$ are at distance at most $\lambda$ from $\left[z, a_{2}\right]$. The first case cannot occur if $\theta>\lambda+\kappa$. In the second case dist $\left(a_{5},\left\{a_{6}, a_{7}\right\}\right) \geq \theta-2 \lambda$. The intersection $\left[a_{3}, a_{5}\right] \cap\left[a_{6}, a_{5}\right]$ has length at least $\theta-2 \sigma-2 \lambda$. By the uniform quasi-convexity of subsets in $\mathcal{A}$ (Qconv) this intersection is contained in $\mathcal{N}_{t \kappa+1}(A) \cap \mathcal{N}_{t \kappa+1}\left(A^{\prime}\right)$. If $\theta>2 \sigma+2 \lambda+\operatorname{diam}_{t \kappa+1}+1$ then $A=A^{\prime}$.

The point $a_{4}$ is the entrance point of $\left[x, b_{3}\right]$ in $\overline{\mathcal{N}}_{\kappa}(A)$ while $a_{6}$ is the entrance point of $\left[a_{3}, z\right]$ in $\overline{\mathcal{N}}_{\kappa}(A)$. If $a_{4} \in\left[a_{3}, b_{3}\right]$ then $a_{4}=a_{6}$, and this point is at distance at most $\lambda$ from both $[x, w]$ and $[y, z]$. If $\theta>2 \lambda$ then this cannot occur. Thus we may assume that $a_{4} \in\left[x, a_{3}\right]$. Likewise we have that $a_{7} \in\left[b_{3}, z\right]$ (see Figure 6).

We apply Lemma 4.32 to $\left[x, a_{1}\right]$ and to $a_{4} \in\left[x, a_{3}\right]$. If we are in the second case of the conclusion then $a_{4} \in \overline{\mathcal{N}}_{\kappa}\left(A^{\prime \prime}\right)$, and the entrance and exit point, $a_{4}^{\prime}, a_{4}^{\prime \prime}$, of $\left[x, a_{3}\right]$ from $\overline{\mathcal{N}}_{\kappa}\left(A^{\prime \prime}\right)$ are at distance at most $\lambda$ from $\left[x, a_{1}\right]$. If $\operatorname{dist}\left(a_{4}, a_{4}^{\prime \prime}\right) \geq \operatorname{diam}_{t \kappa+1}+1$ then $A^{\prime \prime}=A$ and $a_{4}=a_{4}^{\prime}$. Thus, in all cases $a_{4}$ is at distance $O(1)$ from $\left[x, a_{1}\right]$. Recall that $a_{4}$ is at distance at most $\lambda$ from [x,w]. It follows that if $\theta$ is large enough then $a_{4} \in B(x, 2 \theta+\lambda)$.

A similar argument gives that $a_{7} \in B(z, 2 \theta+\lambda)$.

We have thus that $\{x, z\} \subset \overline{\mathcal{N}}_{2 \theta+\lambda+\kappa}(A)$. Also, since $\left\{a_{3}, b_{3}\right\} \subset\left[a_{4}, a_{7}\right] \subset$ $\overline{\mathcal{N}}_{t \kappa}(A)$ it follows that $\{y, w\} \subset \overline{\mathcal{N}}_{2 \theta+2 \sigma+t \kappa}(A)$. The quasi-convexity property (Qconv) applied to $A$ implies that $P \subset \overline{\mathcal{N}}_{\chi}(A)$, where $\chi=t(2 \theta+\lambda+2 \sigma+t \kappa)$.

Case 2. Assume that the triangle $x y z$ has a central piece, i.e., it is in case $(\mathrm{P})$ of Definition 4.27, while $x z w$ has a central point, i.e., it is in case (C). Then there exists $A \in \mathcal{A}$ such that $\overline{\mathcal{N}}_{\sigma}(A)$ intersects all the edges of $x y z$. Moreover if $x_{2}, y_{1}$ are the entrance and exit point of $[x, y]$ in $\overline{\mathcal{N}}_{\sigma}(A)$, while $y_{2}, z_{1}$ and $z_{2}, x_{1}$ are the entranceand exit points of $[y, z]$ and respectively $[z, x]$ in $\overline{\mathcal{N}}_{\sigma}(A)$ then $\operatorname{dist}\left(x_{1}, x_{2}\right)$, $\operatorname{dist}\left(y_{1}, y_{2}\right)$ and $\operatorname{dist}\left(z_{1}, z_{2}\right)$ are at most $\delta$.

Let also $b_{1} \in[z, w], b_{2} \in[w, x]$ and $b_{3} \in[x, z]$ be such that $\left\{b_{1}, b_{2}, b_{3}\right\}$ has diameter at most $2 \sigma$. If $\theta>2 \sigma$ then property (Fat 1 ) implies that $\left\{b_{1}, b_{2}\right\} \subset$ $B(w, 2 \theta)$.

Case 2.a. Assume that $b_{3} \in\left[x_{1}, z_{2}\right]$. Note that $\operatorname{dist}\left(b_{3}, x_{1}\right) \geq \operatorname{dist}(w,[x, y])-2 \theta-$ $2 \sigma-\delta \geq 6 \theta-2 \sigma-\delta$. Same for $\operatorname{dist}\left(b_{3}, z_{2}\right)$. Thus for $\theta$ large both $\operatorname{dist}\left(b_{3}, x_{1}\right)$ and $\operatorname{dist}\left(b_{3}, z_{2}\right)$ are large.

Lemma 4.32 applied to $x_{1} \in\left[x, b_{3}\right]$ and to $\left[x, b_{2}\right]$ implies that either $x_{1} \in$ $\overline{\mathcal{N}}_{\kappa}\left(\left[x, b_{2}\right]\right)$ or $x_{1} \in \overline{\mathcal{N}}_{\kappa}\left(A_{1}\right)$ such that the entrance and exit points $x_{1}^{\prime}, x_{1}^{\prime \prime}$ of $\left[x, b_{3}\right]$ from $\overline{\mathcal{N}}_{\kappa}\left(A_{1}\right)$ are at distance at most $\lambda$ from $\left[x, b_{2}\right]$. In the latter case if $\operatorname{dist}\left(x_{1}, x_{1}^{\prime \prime}\right)>$ $\operatorname{diam}_{\tau}$ where $\tau=t \max (\sigma, \kappa)+1$ then $A_{1}=A$ and $x_{1}=x_{1}^{\prime}$. Thus in all cases $\operatorname{dist}\left(x_{1},[x, w]\right)=O(1)$. For $\theta$ large enough it follows that $x_{2} \in B(x, 2 \theta)$, hence $x \in \overline{\mathcal{N}}_{2 \theta+\sigma}(A)$. A similar argument gives that $z \in \overline{\mathcal{N}}_{2 \theta+\sigma}(A)$. 
If $\theta>\delta$ then $\operatorname{dist}\left(y, y_{1}\right)<2 \theta$ and $y \in \overline{\mathcal{N}}_{2 \theta+\sigma}(A)$.

Also $b_{3} \in\left[x_{1}, z_{2}\right] \subset \overline{\mathcal{N}}_{t \sigma}(A)$, hence $w \in \overline{\mathcal{N}}_{t \sigma+2 \theta+2 \sigma}(A)$. We conclude by the quasi-convexity property (Qconv) that $P \subset \mathcal{N}_{\chi}(A)$ for some $\chi=O(1)$.

Case 2.b. Assume that $b_{3} \notin\left[x_{1}, z_{2}\right]$. Without loss of generality we may assume that $\left[x_{1}, z_{2}\right] \subset\left[x, b_{3}\right)$.

Lemma 4.32 applied to $b_{3} \in\left[z_{2}, z\right]$ and to $\left[z_{1}, z\right]$ implies that either $b_{3} \in$ $\overline{\mathcal{N}}_{\kappa}\left(\left[z_{1}, z\right]\right)$ or that $b_{3} \in \overline{\mathcal{N}}_{\kappa}(B)$ such that $\overline{\mathcal{N}}_{\kappa}(B)$ intersects $\left[z_{1}, z\right]$, and if $b_{4}, b_{5}$ are the entrance and exit point of $\left[z_{2}, z\right]$ from $\overline{\mathcal{N}}_{\kappa}(B)$ then these points are at distance at most $\lambda$ from $\left[z_{1}, z\right]$. For $\theta$ large enough the first case cannot occur. In the second case $\operatorname{dist}\left(b_{3},\left\{b_{4}, b_{5}\right\}\right) \geq \operatorname{dist}(w,[y, z])-\lambda-2(\theta+\sigma) \geq 6 \theta-\lambda-2 \sigma$.

Applying Lemma 4.32 now to $b_{4} \in\left[x, b_{3}\right]$ and to $\left[x, b_{2}\right]$ gives that for $\theta$ large enough $b_{4} \in \overline{\mathcal{N}}_{\kappa}\left(B^{\prime}\right)$ such that $\overline{\mathcal{N}}_{\kappa}\left(B^{\prime}\right)$ intersects $\left[x, b_{3}\right]$, and the entrance and exit points $b_{6}, b_{7}$ of $\left[x, b_{3}\right]$ from $\overline{\mathcal{N}}_{\kappa}\left(B^{\prime}\right)$ are at distance at most $\lambda$ from $\left[x, b_{2}\right]$ (see Figure 7). Moreover dist $\left(b_{4},\left\{b_{6}, b_{7}\right\}\right) \geq \theta-2 \lambda$. Thus $\overline{\mathcal{N}}_{t \kappa}(B) \cap \overline{\mathcal{N}}_{t \kappa}\left(B^{\prime}\right)$ contains $\left[b_{4}, b_{7}\right] \cap\left[b_{4}, b_{3}\right]$ of length $\min (6 \theta-\lambda-2 \sigma, \theta-2 \lambda)$. For $\theta$ large we conclude that $B=B^{\prime}$.

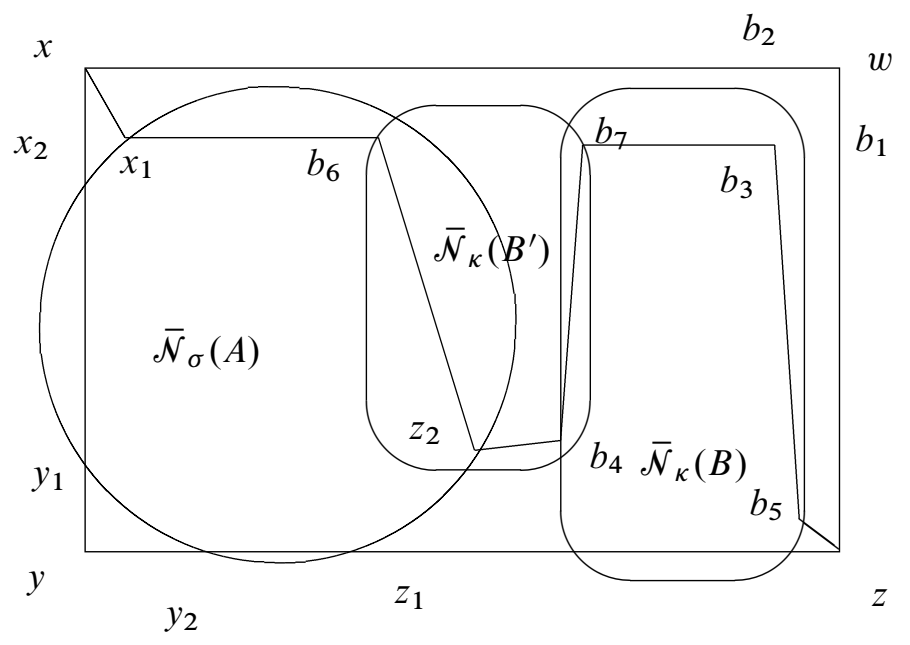

Figure 7. Case 2.B in the proof of Lemma 4.33.

The point $b_{6}$ is the entrance point of $\left[x, b_{3}\right]$ into $\overline{\mathcal{N}}_{\kappa}(B)$ while $b_{4}$ is the entrance point of $\left[z_{2}, z\right]$ into $\overline{\mathcal{N}}_{\kappa}(B)$. If $b_{6} \in\left[z_{2}, b_{3}\right]$ then $b_{6}=b_{4}$ and $\operatorname{dist}([y, z],[x, w]) \leq$ $2 \lambda$. For $\theta$ large enough this case is impossible. Thus $b_{6} \in\left[x, z_{2}\right]$.

The intersection $\left[x_{1}, z_{2}\right] \cap\left[b_{6}, z_{2}\right]$ is in $\overline{\mathcal{N}}_{t \sigma}(A) \cap \overline{\mathcal{N}}_{t \kappa}(B)$. Note that $\operatorname{dist}\left(b_{6}, z_{2}\right) \geq$ $\theta-\lambda-\delta$, thus for $\theta$ large we may assume that $\operatorname{dist}\left(b_{6}, z_{2}\right)>\operatorname{diam}_{\tau}+1$ with $\tau=t \max (\sigma, \kappa)+1$.

If $\operatorname{dist}\left(x_{1}, z_{2}\right) \leq \operatorname{diam}_{\tau}+1$ then $\left\{x_{2}, x_{1}, z_{1}\right\}$ has diameter $O(1)$ and we are back 
in Case 1 with $a_{1}=x_{2}, a_{2}=z_{1}$ and $a_{3}=x_{1}$ and with the constant $\sigma$ possibly larger. We may then use the proof in Case 1 to finish the argument.

Assume now that $\operatorname{dist}\left(x_{1}, z_{2}\right)>\operatorname{diam}_{\tau}+1$. Then $A=B$ and $b_{5}$, the entrance point of $\left[z, z_{2}\right]$ into $\overline{\mathcal{N}}_{\kappa}(B)$, is also the entrance point of $[z, x]$ into $\overline{\mathcal{N}}_{\kappa}(A)$. As $z_{2}$ is the entrance point of $[z, x]$ into $\overline{\mathcal{N}}_{\sigma}(A)$, Lemma 4.31 implies that $\operatorname{dist}\left(z_{2}, b_{5}\right)=O(1)$.

By construction $b_{3} \in\left[z_{2}, b_{5}\right]$, hence $\operatorname{dist}\left(b_{3}, z_{2}\right)=O(1)$. On the other hand $\operatorname{dist}\left(b_{3}, z_{2}\right) \geq \operatorname{dist}(w,[y, z])-2 \sigma-2 \theta-\delta \geq 6 \theta-2 \sigma-\delta$. Thus for $\theta$ large enough we obtain a contradiction.

Case 3. Assume that both geodesic triangles $x y z$ and $x z w$ have central pieces, that is, they are in case (P) of Definition 4.27. Then there exists $A_{1}$ in $\mathcal{A}$ such that $\overline{\mathcal{N}}_{\sigma}\left(A_{1}\right)$ intersects all the edges of $x y z$. Moreover the pairs of entrance points in $\overline{\mathcal{N}}_{\sigma}\left(A_{1}\right)$, $\left(x_{1}, x_{2}\right),\left(y_{1}, y_{2}\right)$ and $\left(z_{1}, z_{2}\right)$ are all at respective distances less than $\delta$. Likewise there exists $A_{2}$ in $\mathcal{A}$ such that $\overline{\mathcal{N}}_{\sigma}\left(A_{2}\right)$ intersects all the edges of $x z w$, and the pairs of entrance points in $\overline{\mathcal{N}}_{\sigma}\left(A_{2}\right),\left(x_{1}^{\prime}, x_{2}^{\prime}\right),\left(z_{1}^{\prime}, z_{2}^{\prime}\right)$ and $\left(w_{1}, w_{2}\right)$ are all at distances less than $\delta$ (see Figure 8).

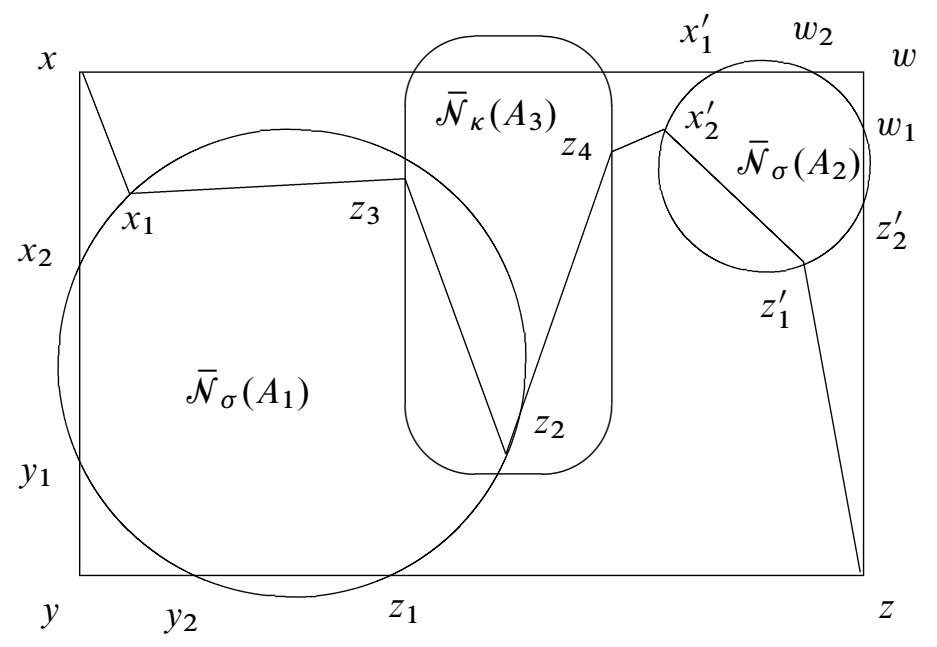

Figure 8. Case 3 in the proof of Lemma 4.33.

If $\theta>\delta$ then $y_{1}, y_{2} \in B(y, 2 \theta)$ and $w_{1}, w_{2} \in B(w, 2 \theta)$.

If $A_{1}=A_{2}=A$ then $x_{1}=x_{2}^{\prime}, z_{2}=z_{1}^{\prime}$, hence $\operatorname{dist}\left(x_{1}^{\prime}, x_{2}\right)$ and $\operatorname{dist}\left(z_{1}, z_{2}^{\prime}\right)$ are less than $2 \delta$. If $\theta>2 \delta$ it follows that $x_{1}^{\prime}, x_{2} \in B(x, 2 \theta)$ and that $z_{1}, z_{2}^{\prime} \in B(z, 2 \theta)$. Thus $x, y, z, w \in \overline{\mathcal{N}}_{2 \theta+\sigma}(A)$, which by (Qconv) implies that $P \subset \mathcal{N}_{\chi}(A)$ for $\chi>t(2 \theta+\sigma)$.

Assume that $A_{1} \neq A_{2}$. Then $\left[x_{1}, z_{2}\right]$ and $\left[x_{2}^{\prime}, z_{1}^{\prime}\right]$ are either disjoint or they intersect in a sub-geodesic of length at most $\operatorname{diam}_{t \sigma+1}$. 
Let $\tau=t \max (\sigma, \kappa)+1$. If either $\left[x_{1}, z_{2}\right]$ or $\left[x_{2}^{\prime}, z_{1}^{\prime}\right]$ is of length at most $\operatorname{diam}_{\tau}+1$ then either $\left\{x_{2}, x_{1}, z_{1}\right\}$ or $\left\{z_{1}^{\prime}, z_{2}^{\prime}, x_{1}^{\prime}\right\}$ is of diameter at most $\operatorname{diam}_{\tau}+1+2 \delta$. Therefore we find ourselves in Case 2.B, with the constant $\sigma$ possibly larger. We can then finish the argument as in that case.

If both $\left[x_{1}, z_{2}\right]$ and $\left[x_{2}^{\prime}, z_{1}^{\prime}\right]$ have length larger than $\operatorname{diam}_{\tau}+1$ and their intersection is non-empty then either $x_{1}^{\prime}$ and $z_{1}$ or $x_{2}$ and $z_{2}^{\prime}$ are at distance at most $2 \delta+\operatorname{diam} m_{t \sigma+1}$. If $\theta>2 \delta+\operatorname{diam}_{t \sigma+1}$ then this is impossible. We may therefore assume that $\left[x_{1}, z_{2}\right]$ and $\left[x_{2}^{\prime}, z_{1}^{\prime}\right]$ do not intersect, and are both of length larger than $\operatorname{diam}_{\tau}+1$.

Without loss of generality we may also assume that $\left[x_{1}, z_{2}\right] \subset\left[x, x_{2}^{\prime}\right)$ holds. Lemma 4.32 applied to the geodesic $\left[x, x_{1}^{\prime}\right]$ and the point $z_{2} \in\left[x, x_{2}^{\prime}\right]$ implies that either $z_{2} \in \overline{\mathcal{N}}_{\kappa}([x, w])$ or $z_{2} \in \overline{\mathcal{N}}_{\kappa}\left(A_{3}\right)$, where $\overline{\mathcal{N}}_{\kappa}\left(A_{3}\right)$ intersects $\left[x, x_{1}^{\prime}\right]$, and the entrance and exit points $z_{3}, z_{4}$ of $\left[x, x_{2}^{\prime}\right]$ in $\overline{\mathcal{N}}_{\kappa}\left(A_{3}\right)$ are at distance at most $\lambda$ from $\left[x, x_{1}^{\prime}\right]$ (see Figure 8). If $\theta>\delta+\kappa$ then the first case cannot occur. In the second case we have that dist $\left(z_{2},\left\{z_{3}, z_{4}\right\}\right)>\theta-\delta-\lambda$. In particular we may assume that $\operatorname{dist}\left(z_{2}, z_{3}\right)>\operatorname{diam}_{\tau}+1$. We also have the assumption that $\operatorname{dist}\left(x_{1}, z_{2}\right)>$ $\operatorname{diam}_{\tau}+1$. Then $\left[x_{1}, z_{2}\right] \cap\left[z_{3}, z_{2}\right]$ has diameter $>\operatorname{diam}_{\tau}$ and it is contained in $\overline{\mathcal{N}}_{t \sigma}\left(A_{1}\right) \cap \overline{\mathcal{N}}_{t \kappa}\left(A_{3}\right)$. Therefore $A_{1}=A_{3}$. In particular $z_{4}$, the exit point of $\left[x, x_{2}^{\prime}\right]$ from $\overline{\mathcal{N}}_{\kappa}\left(A_{1}\right)$, and $z_{2}$, the exit point of $[x, z]$ (therefore also of $\left[x, x_{2}^{\prime}\right]$ ) from $\overline{\mathcal{N}}_{\sigma}\left(A_{1}\right)$ are at distance $O(1)$ by Lemma 4.31. It follows that $z_{1}$ and $[x, w]$ are at distance $O(1)$, and if $\theta$ is large enough this gives a contradiction.

Lemma 4.35. Let $(X$, dist) and $\mathcal{A}$ satisfy the hypotheses of Lemma 4.33. Then in any asymptotic cone of $X$ property $\left(\Pi_{2}\right)$ is satisfied by the collection of limit sets $\mathcal{A}_{\omega}$ (i.e., any simple non-trivial bigon with edges limit geodesics is contained in a subset from $\left.\mathcal{A}_{\omega}\right)$.

Proof. In an asymptotic cone $\operatorname{Con}_{\omega}(X ; e, d)$ consider a simple bigon of endpoints $x, y$ whose edges are limit geodesics. Then there exist two sequences of geodesics $\left[x_{n}, y_{n}\right]$ and $\left[x_{n}^{\prime}, y_{n}^{\prime}\right]$ such that $\omega$-almost surely $\operatorname{dist}\left(x_{n}, x_{n}^{\prime}\right)$ and $\operatorname{dist}\left(y_{n}, y_{n}^{\prime}\right)$ are of order $o\left(d_{n}\right)$, while $\operatorname{dist}\left(x_{n}, y_{n}\right)$ and $\operatorname{dist}\left(x_{n}^{\prime}, y_{n}^{\prime}\right)$ are of order $O\left(d_{n}\right)$. Let $m_{n}$ and $m_{n}^{\prime}$ be the middlepoints of $\left[x_{n}, y_{n}\right]$ and respectively of $\left[x_{n}^{\prime}, y_{n}^{\prime}\right]$. Let $\delta_{n}$ be the maximum between $\operatorname{dist}\left(\left[x_{n}, m_{n}\right],\left[x_{n}^{\prime}, m_{n}^{\prime}\right]\right)$ and $v \theta$, where $\theta$ and $v$ are the constants provided by Lemma 4.33. Then $\delta_{n}={ }_{\omega} o\left(d_{n}\right)$. Similarly, $\delta_{n}^{\prime}=\max \left\{\operatorname{dist}\left(\left[m_{n}, y_{n}\right],\left[m_{n}^{\prime}, y_{n}^{\prime}\right]\right), v \theta\right\}$ satisfies $\delta_{n}^{\prime}={ }_{\omega} o\left(d_{n}\right)$. Let $x_{n}^{1}$ be the farthest from $x_{n}$ point on $\left[x_{n}, m_{n}\right]$ at distance $\delta_{n}$ from $\left[x_{n}^{\prime}, m_{n}^{\prime}\right]$, and let $x_{n}^{2}$ be the farthest from $x_{n}^{\prime}$ point on $\left[x_{n}^{\prime}, m_{n}^{\prime}\right]$ at distance $\delta_{n}$ from $x_{n}^{1}$. We choose in a similar manner $y_{n}^{1} \in\left[y_{n}, m_{n}\right]$ and $y_{n}^{2} \in\left[y_{n}^{\prime}, m_{n}^{\prime}\right]$. Since the limit bigon is simple, it follows that the sets $\left\{x_{n}, x_{n}^{\prime}, x_{n}^{1}, x_{n}^{2}\right\}$ and $\left\{y_{n}, y_{n}^{\prime}, y_{n}^{1}, y_{n}^{2}\right\}$ have diameters of order $o\left(d_{n}\right) \omega$-almost surely.

We prove that any quadrilateral having as two opposite edges $\left[x_{n}^{1}, y_{n}^{1}\right] \subset\left[x_{n}, y_{n}\right]$ and $\left[x_{n}^{2}, y_{n}^{2}\right] \subset\left[x_{n}^{\prime}, y_{n}^{\prime}\right]$ is $(\theta, v)$-fat. This suffices to finish the argument, making use of Lemma 4.33. 
$\left(\right.$ Fat $\left._{1}\right)$ : By construction dist $\left(\left[x_{n}^{1}, y_{n}^{1}\right],\left[x_{n}^{2}, y_{n}^{2}\right]\right) \geq v \theta$, while the edges $\left[x_{n}^{1}, x_{n}^{2}\right]$ and $\left[y_{n}^{1}, y_{n}^{2}\right]$ are at distance $O\left(d_{n}\right)$ from each other. The rest of the property follows by Lemma 4.7.

Property $\left(\right.$ Fat $\left._{2}\right)$ follows immediately from the fact that $\operatorname{dist}\left(\left[x_{n}^{1}, y_{n}^{1}\right],\left[x_{n}^{2}, y_{n}^{2}\right]\right) \geq$ $\nu \theta$ and that $\operatorname{dist}\left(\left[x_{n}^{1}, x_{n}^{2}\right],\left[y_{n}^{1}, y_{n}^{2}\right]\right)=O\left(d_{n}\right)$.

The following statement ends the proof of Theorem 4.30.

Lemma 4.36. Let $(X$, dist) be $(*)$-ATG with respect to $\mathcal{A}$. If $(X, \mathcal{A})$ moreover satisfy properties $\left(\alpha_{1}\right)$ and $\left(\alpha_{2}\right)$ then in any asymptotic cone of $X$ property $\left(\Pi_{3}\right)$ is satisfied.

Proof. Let $\operatorname{Con}_{\omega}(X ; e, d)$ be an arbitrary asymptotic cone of $X$ and let $\mathcal{A}_{\omega}$ be the collection of limit sets of sequences from $\mathcal{A}$. Since $\left(\alpha_{1}\right) \&\left(\alpha_{2}\right) \Rightarrow(\mathrm{Qconv})$ it follows that the sets in $\mathcal{A}_{\omega}$ are geodesic. Also $\left(\alpha_{1}\right) \&\left(\alpha_{2}\right)$ imply that distinct limit sets from $\mathcal{A}_{\omega}$ intersect in at most one point, i.e. property $\left(T_{1}\right)$.

Let $\Delta$ be a non-trivial simple geodesic triangle in $\operatorname{Con}_{\omega}(X ; e, d)$, whose edges $[x, y],[y, z]$ and $[z, x]$ appear as limits of sequences $\left[x_{n}, y_{n}^{\prime}\right],\left[y_{n}, z_{n}^{\prime}\right]$ and $\left[z_{n}, x_{n}^{\prime}\right]$ of geodesics in $X$. Then $\omega$-almost surely $\operatorname{dist}\left(x_{n}, x_{n}^{\prime}\right), \operatorname{dist}\left(y_{n}, y_{n}^{\prime}\right)$ and $\operatorname{dist}\left(z_{n}, z_{n}^{\prime}\right)$ are of order $o\left(d_{n}\right)$, while the lengths of $\left[x_{n}, y_{n}^{\prime}\right],\left[y_{n}, z_{n}^{\prime}\right]$ and $\left[z_{n}, x_{n}^{\prime}\right]$ are of order $O\left(d_{n}\right)$. Let $T_{n}$ be a geodesic triangle with vertices $x_{n}, y_{n}, z_{n}$. We denote its edges by $[u, v]$, with $u, v \in\left\{x_{n}, y_{n}, z_{n}\right\}$. The three limit geodesics $\mathfrak{g}^{x}=\lim _{\omega}\left(\left[y_{n}, z_{n}\right]\right), \mathfrak{g}^{y}=$ $\lim _{\omega}\left(\left[x_{n}, z_{n}\right]\right)$ and $\mathrm{g}^{z}=\lim _{\omega}\left(\left[x_{n}, y_{n}\right]\right)$ compose the limit triangle $T=\lim _{\omega}\left(T_{n}\right)$.

Case 1. Assume that $\omega$-almost surely the triangle $T_{n}$ is in case (C) of Definition 4.27. Then there exists $a_{n}^{1} \in\left[x_{n}, y_{n}\right], a_{n}^{2} \in\left[y_{n}, z_{n}\right]$ and $a_{n}^{3} \in\left[z_{n}, x_{n}\right]$ such that the set $\left\{a_{n}^{1}, a_{n}^{2}, a_{n}^{3}\right\}$ has $\omega$-almost surely diameter at most $2 \sigma$ for some constant $\sigma$. It follows that $\lim _{\omega}\left(a_{n}^{1}\right)=\lim _{\omega}\left(a_{n}^{2}\right)=\lim _{\omega}\left(a_{n}^{3}\right)=a$. The point $a$ is on the three edges of $T$.

Without loss of generality we may assume that $a \notin\{x, y\}$. The fact that $a \neq x$ implies that either $\mathrm{g}^{z} \neq[x, y]$ or $\mathfrak{g}^{y} \neq[x, z]$. Property $\left(\Pi_{2}\right)$ implies that we may apply Proposition 3.9 to $\mathscr{L}_{1}=[x, y], \mathscr{L}_{2}=[x, z], \mathfrak{g}_{1}=\mathrm{g}^{z}, \mathrm{~g}_{2}=\mathrm{g}^{y}$ and to the intersection point $a \in \mathfrak{g}^{z} \cap \mathfrak{g}^{y}$. We conclude that the $\mathcal{T}$-bigon formed by $\mathfrak{g}^{z}$ and $\mathfrak{g}^{y}$ of endpoints $a, x$ is contained in a subset $A_{x} \in \mathcal{A}_{\omega}$.

Similarly we deduce that the $\mathcal{T}$-bigon formed by $\mathrm{g}^{z}$ and $\mathrm{g}^{x}$ of endpoints $a, y$ is contained in a subset $A_{y} \in \mathcal{A}_{\omega}$.

If $a=z$ then $\mathfrak{g}_{x} \subset A_{y}, \mathfrak{g}_{y} \subset A_{x}$. Also $a \notin[x, y]$, which by Lemma 3.7 implies that $a$ is contained in the interior of a simple $\mathcal{T}$-bigon formed by $[x, y]$ and $\mathrm{g}^{z}$. By property $\left(\Pi_{2}\right)$ this $\mathcal{T}$-bigon is contained in some $A \in \mathcal{A}_{\omega}$. The intersections $A \cap A_{x}$ and $A \cap A_{y}$ contain non-trivial sub-arcs of $\mathrm{g}^{z}$ therefore by $\left(T_{1}\right)$ we conclude that $A=A_{x}=A_{y}$. The subset $A$ contains also $\mathrm{g}^{z}$.

Property $\left(\Pi_{2}\right)$ allows us to apply Lemma 3.8 to the pairs of $\operatorname{arcs}\left(\mathrm{g}^{x},[y, z]\right)$, $\left(\mathfrak{g}^{y},[x, z]\right)$ and $\left(\mathfrak{g}^{z},[x, y]\right)$ and deduce that $\Delta=[x, y] \cup[y, z] \cup[z, x]$ is contained in $A$. 
If $a \neq z$ then again by Proposition 3.9 the $\mathcal{T}$-bigon formed by $\mathfrak{g}^{x}$ and $\mathfrak{g}^{y}$ of endpoints $a, z$ is contained in a subset $A_{z} \in \mathcal{A}_{\omega}$. Since $a$ is not a vertex in $\Delta$ it is contained in at most one edge of $\Delta$. Without loss of generality we assume that $a \notin[x, y] \cup[y, z]$.

The fact that $a \notin[x, y]$, Lemma 3.7, properties $\left(\Pi_{2}\right)$ and $\left(T_{1}\right)$ imply as above that $A_{x}=A_{y}$. Likewise from $a \notin[y, z]$ we deduce that $A_{y}=A_{z}$. Thus $A=A_{x}=$ $A_{y}=A_{z}$ contains $T$. Property $\left(\Pi_{2}\right)$ and Lemma 3.8 imply that $\Delta$ is also contained in $A$.

Case 2. Assume that $\omega$-almost surely $T_{n}$ is in case (P) of Definition 4.27. Then there exist $A_{n}$ in $\mathcal{A}$ such that $\overline{\mathcal{N}}_{\sigma}\left(A_{n}\right)$ intersects all the edges of $T_{n}$. Moreover if $\left(x_{n}^{2}, y_{n}^{1}\right)$, $\left(y_{n}^{2}, z_{n}^{1}\right)$ and $\left(z_{n}^{2}, x_{n}^{1}\right)$ are the pairs of entrance and exit points from $\overline{\mathcal{N}}_{\sigma}(A)$ of $\left[x_{n}, y_{n}\right]$, $\left[y_{n}, z_{n}\right]$ and $\left[z_{n}, x_{n}\right]$ respectively, then $\operatorname{dist}\left(x_{n}^{1}, x_{n}^{2}\right), \operatorname{dist}\left(y_{n}^{1}, y_{n}^{2}\right)$ and $\operatorname{dist}\left(z_{n}^{1}, z_{n}^{2}\right)$ are less than $\delta$.

Let $x^{\prime}=\lim _{\omega}\left(x_{n}^{1}\right)=\lim _{\omega}\left(x_{n}^{2}\right), y^{\prime}=\lim _{\omega}\left(y_{n}^{1}\right)=\lim _{\omega}\left(y_{n}^{2}\right)$ and $z^{\prime}=$ $\lim _{\omega}\left(z_{n}^{1}\right)=\lim _{\omega}\left(z_{n}^{2}\right)$.

Assume that $\left\{x^{\prime}, y^{\prime}, z^{\prime}\right\}$ has cardinality at most 2. Assume for instance that $x^{\prime}=$ $y^{\prime}$. Then the point $a=x^{\prime}=y^{\prime}$ is in $\mathrm{g}^{x} \cap \mathrm{g}^{y} \cap \mathrm{g}^{z}$. With the same argument as in Case 1 we deduce that both $T$ and $\Delta$ are contained in some $A \in \mathcal{A}_{\omega}$.

Assume now that $\left\{x^{\prime}, y^{\prime}, z^{\prime}\right\}$ has cardinality 3 . The geodesic triangle $T^{\prime}$ of vertices $x^{\prime}, y^{\prime}, z^{\prime}$ and with edges contained in the edges of $T$ is included in the piece $A=$ $\lim _{\omega}\left(A_{n}\right)$.

Proposition 3.9 implies that the $\mathcal{T}$-bigon of endpoints $x, x^{\prime}$ formed by $\mathrm{g}^{z}$ and $\mathrm{g}^{y}$ is either trivial or contained in some $A_{x} \in \mathcal{A}_{\omega}$. Similarly, the $\mathcal{T}$-bigon of endpoints $y, y^{\prime}$ formed by $\mathrm{g}^{z}$ and $\mathrm{g}^{x}$ is either trivial or in some $A_{y}$, and the $\mathcal{T}$-bigon of endpoints $z, z^{\prime}$ formed by $\mathrm{g}^{x}$ and $\mathrm{g}^{y}$ is either trivial or in some $A_{z}$.

If $x^{\prime} \neq x$ then $x^{\prime}$ cannot be contained both in $[x, y]$ and in $[x, z]$. Suppose that $x^{\prime} \notin[x, y]$. Then $x^{\prime}$ is in the interior of a non-trivial simple $\mathcal{T}$-bigon formed by $\mathrm{g}^{z}$ and $[x, y]$. This $\mathcal{T}$-bigon is contained in some $B_{x} \in \mathcal{A}_{\omega}$ by $\left(\Pi_{2}\right)$, and its intersections with $A_{x}$ and with $A$ contain a non-trivial sub-arc of $\mathrm{g}^{z}$. Hence $A_{x}=B_{x}=A$. Thus the $\mathcal{T}$-bigon of endpoints $x, x^{\prime}$ is contained in $A$.

In the same way we obtain that the $\mathcal{T}$-bigons of endpoints $y, y^{\prime}$ and $z, z^{\prime}$ are contained in $A$. Thus in all cases $T \subset A$, which by Lemma 3.8 implies that $\Delta \subset A$.

\section{Quasi-isometric rigidity of relatively hyperbolic groups}

In this section we prove one of our main results: that if a group is asymptotically tree-graded then it is relatively hyperbolic (Theorem 5.4). We begin by proving an intermediate result: if a group has an equivariant asymptotically tree-graded structure then it is relatively hyperbolic. 
Proposition 5.1 (equivariant ATG structure implies relative hyperbolicity). Consider a finitely generated group endowed with a word metric ( $G$, dist), which is ATG with respect to a collection of subsets $\mathscr{B}$. Assume moreover that $G$ permutes the subsets in $B$.

Then $G$ is either hyperbolic, or hyperbolic relative to a family of subgroups $\left\{H_{1}, \ldots, H_{m}\right\}$ such that for each $H_{i}$ there exists a unique $B_{i} \in \mathscr{B}$ satisfying $H_{i} \subset$ $B_{i} \subset \mathcal{N}_{\mathcal{K}}\left(H_{i}\right)$, where $\mathcal{K}$ is a constant depending only on $(G$, dist) and $\mathscr{B}$.

Proof. Step 1 . We first prove that only finitely many subsets in $\mathscr{B}$ may contain 1 .

According to the quasi-convexity property (Qconv) of $\mathcal{B}$ (see Theorem 4.22) there exists $\tau>0$ such that for any $x, y$ in some $B \in \mathscr{B}$ any geodesic $[x, y]$ is contained in $\mathcal{N}_{\tau}(B)$. Property $\left(\alpha_{1}\right)$ for $\mathcal{B}$ implies that there exists $D_{\tau}$ such that for $B \neq B^{\prime}$, $\mathcal{N}_{\tau}(B) \cap \mathcal{N}_{\tau}\left(B^{\prime}\right)$ has diameter at most $D_{\tau}$.

Assume that $B \in \mathcal{B}$ contains 1 and has diameter at most $3 D_{\tau}$. Then $B \subset$ $\bar{B}\left(1,3 D_{\tau}\right)$. As $\bar{B}\left(1,3 D_{\tau}\right)$ is finite, only finitely many $B \in \mathscr{B}$ can be in this case.

Assume that $B$ contains 1 and has diameter larger than $3 D_{\tau}$. Then $B$ contains some point $x$ with $\operatorname{dist}(1, x)>3 D_{\tau}$. The geodesic $[1, x]$ is contained in $\mathcal{N}_{\tau}(B)$ and it intersects the sphere around 1 of radius $2 D_{\tau}, S\left(1,2 D_{\tau}\right)$. We define a map from the set $\left\{B \in \mathscr{B} \mid 1 \in B\right.$, diam $\left.B>3 D_{\tau}\right\}$ to the set of subsets of $S\left(1,2 D_{\tau}\right)$, associating to each $B$ the non-empty intersection $\mathcal{N}_{\tau}(B) \cap S\left(1,2 D_{\tau}\right)$. By $\left(\alpha_{1}\right)$ and the choice of $D_{\tau}$, two distinct subsets $B, B^{\prime}$ have disjoint images by the above map, in particular the map is injective. Since the set of subsets of $S\left(1,2 D_{\tau}\right)$ is finite, so is the considered subset of $\mathscr{B}$.

Notation 5.2. Let $\mathcal{F}=\left\{B_{1}, B_{2}, \ldots, B_{k}\right\}$ be the set of $B \in \mathscr{B}$ containing 1 . For every $i \in\{1,2, \ldots, k\}$ let

$$
\mathcal{I}_{i}=\left\{j \in\{1,2, \ldots, k\} \mid g B_{i}=B_{j} \text { for some } g \in G\right\} .
$$

For every $j \in \mathcal{I}_{i}$ we fix $g_{j} \in G$ such that $g_{j} B_{i}=B_{j}$.

Define the constants $\mathcal{K}_{i}=\max _{j \in \mathcal{I}_{i}} \operatorname{dist}\left(1, g_{j}\right)$ and $\mathcal{K}=\max _{1 \leq i \leq k} \mathcal{K}_{i}$.

Step 2. We show that for every $B \in \mathcal{B}$ the stabilizer Stab $(B)=\{g \in G \mid g B=B\}$ is a subgroup of $G$ acting $\mathcal{K}$-transitively on $B$ (in the sense of Definition 2.1).

Let $x$ and $b$ be arbitrary points in $B$. Both subsets $b^{-1} B$ and $x^{-1} B$ contain 1 and are in $\mathcal{B}$. It follows that $b^{-1} B=B_{i}$ and $x^{-1} B=B_{j}$ for some $i, j \in\{1,2, \ldots, k\}$. Since $b^{-1} \times B_{j}=B_{i}$ it follows that $j \in \mathcal{I}_{i}$ and that $B_{j}=g_{j} B_{i}$. The last equality can be re-written as $x^{-1} B=g_{j} b^{-1} B$ which implies that $x g_{j} b^{-1} \in \operatorname{Stab}(B)$, hence that $x$ is at distance at most $\operatorname{dist}\left(1, g_{j}\right)$ from $\operatorname{Stab}(B) b$. This finishes the proof of the fact that $\operatorname{Stab}(B)$ acts $\mathcal{K}$-transitively on $B$.

In particular we have that

$$
\operatorname{Stab}\left(B_{i}\right) \subset B_{i} \subset \mathcal{N}_{\mathcal{K}}\left(\operatorname{Stab}\left(B_{i}\right)\right) \text { for all } i \in\{1,2, \ldots, k\} .
$$


Let $\operatorname{diam}_{2} \mathcal{K}$ be the uniform bound given by property $\left(\alpha_{1}\right)$ for $(G, \mathscr{B})$ and $\delta=2 \mathcal{K}$ (see Notation 4.11).

If all the subsets in $\mathscr{B}$ have diameter at most $\operatorname{diam}_{2} \mathcal{K}+1$ then $G$ is hyperbolic by Corollary 4.21. Thus, in what follows we may assume that $\mathcal{B}$ contains subsets of diameter larger than $\operatorname{diam}_{2 \mathcal{K}}+1$.

Notation 5.3. Denote by $\mathscr{B}^{\prime}$ the set of $B \in \mathscr{B}$ of diameter larger than $\operatorname{diam}_{2} \mathcal{K}+1$.

Let $\widetilde{F}^{\prime}=\mathscr{F} \cap \mathscr{B}^{\prime}$. Let $\mathscr{F}_{0}$ be a subset of $\mathscr{F}^{\prime}$ such that for every $B \in \mathscr{F}^{\prime}$, its orbit $G \cdot B$ intersects $\mathcal{F}_{0}$ in a unique element (such a subset can be obtained for instance by considering one by one the elements $B_{i}$ in $\mathcal{F}^{\prime}$, and deleting from $\mathcal{F}^{\prime}$ all $B_{j}$ with $j \in \mathcal{I}_{i}, j \neq i$ ). It follows that for every $B \in \mathscr{B}^{\prime}$, the orbit $G \cdot B$ intersects $\mathcal{F}_{0}$ in only one element.

Let $\bar{B}_{1}, \ldots, \bar{B}_{m}$ be the elements of $\mathcal{F}_{0}$.

Corollary 4.26 implies that $G$ is ATG with respect to $\mathscr{B}^{\prime}$. Obviously $G$ also permutes the subsets in $\mathscr{B}^{\prime}$.

Step 3. We prove that for every $B \in \mathscr{B}^{\prime}$ there exists a unique $j \in\{1,2, \ldots, m\}$ and a unique left coset $g \operatorname{Stab}\left(\bar{B}_{j}\right)$ such that

$$
g \operatorname{Stab}\left(\bar{B}_{j}\right) \subset B \subset \mathcal{N}_{\mathcal{K}}\left(g \operatorname{Stab}\left(\bar{B}_{j}\right)\right) .
$$

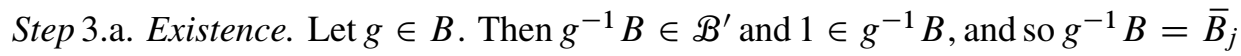
for some $j \in\{1,2, \ldots, m\}$. The double inclusion (3) implies the double inclusion (4). Step 3.b. Unicity. Assume that $g \operatorname{Stab}\left(\bar{B}_{j}\right)$ and $g^{\prime} \operatorname{Stab}\left(\bar{B}_{l}\right)$ both satisfy (4), for $j, l \in\{1,2, \ldots, m\}$. Then

$$
g \bar{B}_{j} \subset \mathcal{N}_{\mathcal{K}}\left(g \operatorname{Stab}\left(\bar{B}_{j}\right)\right) \subset \mathcal{N}_{\mathcal{K}}(B) \subset \mathcal{N}_{2} \mathcal{K}\left(g^{\prime} \operatorname{Stab}\left(\bar{B}_{l}\right)\right) \subset \mathcal{N}_{2} \mathcal{K}\left(g^{\prime} \bar{B}_{l}\right) .
$$

Both $g \bar{B}_{j}$ and $g^{\prime} \bar{B}_{l}$ are in $\mathscr{B}^{\prime}$, in particular $g \bar{B}_{j}$ has diameter at least diam $2 \mathcal{K}+1$. Property $\left(\alpha_{1}\right)$ implies that $g \bar{B}_{j}=g^{\prime} \bar{B}_{l}$. According to the definition of $\mathcal{F}_{0}$ this can only happen if $j=l$. Then $g^{-1} g^{\prime}$ is in $\operatorname{Stab}\left(\bar{B}_{j}\right)$, and $g^{\prime} \operatorname{Stab}\left(\bar{B}_{l}\right)$ coincides with $g \operatorname{Stab}\left(\bar{B}_{j}\right)$.

Step 4. We prove that the group $G$ is hyperbolic relative to $\left\{H_{1}, \ldots, H_{m}\right\}$, where $H_{j}=\operatorname{Stab}\left(\bar{B}_{j}\right)$.

The fact that $G$ is ATG with respect to $\mathscr{B}^{\prime}$, Step 3 and Remark 4.2, (2), imply that $G$ is ATG with respect to $\left\{g H_{j} \mid g \in G / H_{j}, j \in\{1,2, \ldots, m\}\right\}$. In particular by (Qconv) each $H_{j}$ is quasi-convex in $G$, hence each $H_{j}$ is finitely generated. Theorem 1.5 implies that $G$ is hyperbolic relative to $H_{1}, \ldots, H_{m}$.

If $G=H_{j}=\operatorname{Stab}\left(\bar{B}_{j}\right)$ then (3) implies that $G=\bar{B}_{j}$.

We are now ready to prove that the existence of an asymptotically tree-graded structure on a group implies relative hyperbolicity. 
Theorem 5.4. Let $(G$, dist) be an infinite finitely generated group endowed with a word metric, which is asymptotically tree-graded with respect to a collection $\mathcal{A}$ of subsets of $G$.

Then the group $G$ is either hyperbolic or relatively hyperbolic with respect to a family of subgroups $\left\{H_{1}, \ldots, H_{m}\right\}$, such that each $H_{i}$ is contained in $\mathcal{N}_{\varkappa}\left(A_{i}\right)$ for some $A_{i} \in \mathcal{A}$, where $\mathcal{x}$ is a constant depending only on ( $G$, dist) and $\mathcal{A}$.

Remark 5.5. If $G$ is hyperbolic then it is hyperbolic relative to $H=\{1\}$. Still, in this case one cannot state that $H$ is contained in some $\mathcal{N}_{\varkappa}(A)$ with $A \in \mathcal{A}$, because in the definition that we adopt of asymptotically tree-graded metric spaces the finite radius tubular neighborhoods of sets $A \in \mathcal{A}$ do not cover the whole space (see Remark 4.12).

Remark 5.6. The constant $\varkappa$ in Theorem 5.4 can be taken to be the maximum between the constant $M$ in property $\left(\beta_{2}\right)$ (formulated in Theorem 4.22), and the constant $\chi$ in property $\left(\beta_{3}\right)$ (formulated in Section 4.2$)$ of $(G, \mathcal{A})$.

Proof. In view of Proposition 5.1, our goal is to construct a new collection of subsets $\mathscr{B}$ with respect to which the group $G$ is ATG, and which is moreover $G$-equivariant.

Step 1 . We begin by constructing the collection $\mathscr{B}$.

The pair $(G, \mathcal{A})$ satisfies properties $\left(\alpha_{1}\right),\left(\beta_{2}\right)$ and $\left(\beta_{3}\right)$. By Corollary 4.21 , if for $\theta>0$ and $v \geq 8$ from $\left(\beta_{3}\right)$ either there exists no $(\theta, v)$-fat geodesic hexagon in the Cayley graph of $G$, or the $(\theta, v)$-fat geodesic hexagons have uniformly bounded diameter, then $G$ is hyperbolic.

Assume from now on that for every $\eta>0$ there exists a $(\theta, v)$-fat geodesic hexagon of diameter at least $\eta$.

For $\mathcal{\varkappa}$ as in Remark 5.6 and $\operatorname{diam}_{\varkappa}$ given by property $\left(\alpha_{1}\right)$ of $\mathcal{A}$ (according to Notation 4.11), consider the set

$$
\Phi=\left\{P \text { geodesic hexagon } \mid P \text { is }(\theta, v) \text {-fat, } \operatorname{diam}(P) \geq \operatorname{diam}_{\mathcal{H}}+1\right\} .
$$

Let $g$ be an arbitrary element in the group $G$. The metric space ( $G$, dist) is asymptotically tree-graded with respect to the collection of subsets $g \mathcal{A}=\{g A \mid A \in \mathcal{A}\}$, moreover the constants in the properties $\left(\alpha_{1}\right),\left(\beta_{2}\right)$ and $\left(\beta_{3}\right)$ for $g \mathcal{A}$ are the same as for $\mathcal{A}$.

Let $P \in \Phi$. Then $P$ is contained in $\mathcal{N}_{\varkappa}(g A)$ for some $A \in \mathcal{A}$. If $P$ is also contained in $\mathcal{N}_{\varkappa}\left(g A^{\prime}\right)$ for $A^{\prime} \in \mathcal{A}$ then $\mathcal{N}_{\varkappa}(A) \cap \mathcal{N}_{\varkappa}\left(A^{\prime}\right)$ has diameter at least the diameter of $P$, hence at least $\operatorname{diam}_{\varkappa}+1$, consequently $A=A^{\prime}$. Thus $P$ defines a map

$$
\mathbf{A}_{P}: G \rightarrow \mathcal{A}, \quad \mathbf{A}_{P}(g)=A \text { such that } P \subset \mathcal{N}_{\varkappa}(g A) .
$$

We may then define

$$
\mathbf{A}: \Phi \rightarrow \operatorname{Map}(G, \mathcal{A}), \quad \mathbf{A}(P)=\mathbf{A}_{P},
$$


where $\operatorname{Map}(G, \mathcal{A})$ is the set of maps from $G$ to $\mathcal{A}$. Consider the equivalence relation on $\Phi$ induced by $\mathbf{A}$, that is

$P \sim P^{\prime} \Longleftrightarrow \mathbf{A}(P)=\mathbf{A}\left(P^{\prime}\right) \Longleftrightarrow P$ and $P^{\prime}$ are in the same $\mathcal{N}_{\varkappa}(g A)$ for all $g \in G$.

Let $[P]$ be the equivalence class of a hexagon $P$ in $\Phi$. To it we associate the set

$$
B[P]=\bigcap_{g \in G} \mathcal{N}_{\varkappa}\left(g \mathbf{A}_{P}(g)\right) .
$$

We consider the whole collection of such sets

$$
\mathscr{B}=\{B[P] \mid[P] \in \Phi / \sim\} .
$$

Step 2. We prove that ( $G$, dist) is ATG with respect to $\mathcal{B}$.

According to Proposition 4.24 it suffices to prove that $(G, \mathscr{B})$ satisfy property $\left(\alpha_{1}\right)$ from Theorem 4.9 , property $\left(\beta_{2}\right)$ from Theorem 4.22 , and property $\left(\beta_{3}^{\eta}\right)$ from Proposition 4.24, for some $\eta>0$. The proof relies on the simple remark that for every $r>0$,

$$
\mathcal{N}_{r}(B[P]) \subset \bigcap_{g \in G} \mathcal{N}_{r+\varkappa}\left(g \mathbf{A}_{P}(g)\right) .
$$

We begin by proving property $\left(\alpha_{1}\right)$ on the uniformly bounded diameter of intersections of tubular neighborhoods of distinct subsets in $\mathcal{B}$. Let $[P] \neq\left[P^{\prime}\right]$, which is equivalent to the fact that there exists $g_{0} \in G$ such that $P \subset \mathcal{N}_{\varkappa}\left(g_{0} A\right)$ and $P^{\prime} \subset \mathcal{N}_{\varkappa}\left(g_{0} A^{\prime}\right)$ with $A \neq A^{\prime}$. For every $\delta>0$,

$$
\begin{aligned}
\mathcal{N}_{\delta}(B[P]) \cap \mathcal{N}_{\delta}\left(B\left[P^{\prime}\right]\right) & \subset \mathcal{N}_{\delta+\varkappa}\left(g_{0} A\right) \cap \mathcal{N}_{\delta+\varkappa}\left(g_{0} A^{\prime}\right) \\
& =g_{0}\left[\mathcal{N}_{\delta+\varkappa}(A) \cap \mathcal{N}_{\delta+\varkappa}\left(A^{\prime}\right)\right] .
\end{aligned}
$$

Property $\left(\alpha_{1}\right)$ for $\mathcal{A}$ implies that the diameter of $\mathcal{N}_{\delta}(B[P]) \cap \mathcal{N}_{\delta}\left(B\left[P^{\prime}\right]\right)$ is uniformly bounded.

We now prove $\left(\beta_{2}\right)$. Let $\epsilon$ be the constant appearing in $\left(\beta_{2}\right)$ for $\mathcal{A}$. Take $\epsilon^{\prime}=\frac{\epsilon}{2}$ and take $M^{\prime}=\frac{\epsilon+1}{\epsilon} \varkappa$. We prove that $\left(\beta_{2}\right)$ holds for $\mathcal{B}$ with the constants $\epsilon^{\prime}$ and $M^{\prime}$.

Let $g$ be a geodesic of length $\ell$ and let $[P] \in \Phi / \sim$ be such that $g(0)$ and $g(\ell)$ are in $\mathcal{N}_{\epsilon^{\prime} \ell}(B[P])$. It follows that for every $g \in G, g(0)$ and $g(\ell)$ are in $\mathcal{N}_{\epsilon^{\prime} \ell+\varkappa}\left(g \mathbf{A}_{P}(g)\right)$.

If $\varkappa \geq \frac{\epsilon}{2} \ell \Leftrightarrow \ell \leq \frac{2 \varkappa}{\epsilon}$ then $g \subset \overline{\mathcal{N}}_{\frac{\varkappa}{\epsilon}}(\{\mathrm{g}(0), \mathfrak{g}(\ell)\}) \subset \mathcal{N}_{\frac{\varkappa}{\epsilon}+\varkappa}(B[P])=$ $\mathcal{N}_{M^{\prime}}(B[P])$.

Assume that $\varkappa<\frac{\epsilon}{2} \ell$. Then for every $g \in G$ the geodesic $g^{-1} \mathfrak{g}$ of length $\ell$ has its endpoints in $\mathcal{N}_{\epsilon \ell}\left(\mathbf{A}_{P}(g)\right)$. Property $\left(\beta_{2}\right)$ implies that $g^{-1} \mathfrak{g}\left(\left[\frac{\ell}{3}, \frac{2 \ell}{3}\right]\right)$ is contained in $\mathcal{N}_{M}\left(\mathbf{A}_{P}(g)\right) \subset \mathcal{N}_{\varkappa}\left(\mathbf{A}_{P}(g)\right)$.

We have thus obtained that $g\left(\left[\frac{\ell}{3}, \frac{2 \ell}{3}\right]\right)$ is contained in $\mathcal{N}_{\mathcal{\varkappa}}\left(g \mathbf{A}_{P}(g)\right)$ for every $g \in G$. It follows that $g\left(\left[\frac{\ell}{3}, \frac{2 \ell}{3}\right]\right)$ is contained in $B[P]$. 
Property $\left(\beta_{3}^{\eta}\right)$ holds for the constants $\theta$ and $v$ same as in $\left(\beta_{3}\right)$ for $\mathcal{A}$, for the constant $\chi$ equal to 0 , and $\eta=\operatorname{diam}_{\mathcal{\varkappa}}+1$. Indeed every $P \in \Phi$ is contained in $B[P]$.

Step 3. We now show that the group $G$ permutes the subsets in $\mathcal{B}$.

First we note that if $P \sim P^{\prime}$ then $g P \sim g P^{\prime}$ for every $g \in G$. Consequently $G$ acts on the left on $\Phi / \sim$.

Indeed, the set $\mathbf{A}_{P}(\gamma)$ is defined by the inclusion $P \subset \mathcal{N}_{\varkappa}\left(\gamma \mathbf{A}_{P}(\gamma)\right)$. For every $g \in G, g P \subset \mathcal{N}_{\varkappa}\left(g \gamma \mathbf{A}_{P}(\gamma)\right)$, hence $\mathbf{A}_{g P}(g \gamma)=\mathbf{A}_{P}(\gamma)$. From this can be deduced that $P \sim P^{\prime} \Rightarrow g P \sim g P^{\prime}$.

Next we prove that for every $g \in G$ and $P \in \Phi, g B[P]=B[g P]$, thereby ending the proof of the fact that $G$ permutes the subsets in $\mathscr{B}$.

The translate $g B[P]=\bigcap_{\gamma \in G} \mathcal{N}_{\varkappa}\left(g \gamma \mathbf{A}_{P}(\gamma)\right)$ is equal to

$$
\bigcap_{\gamma \in G} \mathcal{N}_{\varkappa}\left(g \gamma \mathbf{A}_{g P}(g \gamma)\right)=\bigcap_{\gamma^{\prime} \in G} \mathcal{N}_{\varkappa}\left(\gamma^{\prime} \mathbf{A}_{g P}\left(\gamma^{\prime}\right)\right)=B[g P]
$$

Proposition 5.1 and the statements proved in Steps 2 and 3 imply the conclusion of Theorem 5.4.

An important consequence of Theorem 5.4 is the following.

Theorem 5.7. Let $G$ be a group hyperbolic relative to a family of subgroups $\mathcal{H}=$ $\left\{H_{1}, \ldots, H_{n}\right\}$. If a group $G^{\prime}$ is $(L, C)$-quasi-isometric to $G$ then $G^{\prime}$ is hyperbolic relative to $\mathscr{H}^{\prime}=\left\{H_{1}^{\prime}, \ldots, H_{m}^{\prime}\right\}$, where each $H_{i}^{\prime}$ can be embedded $(\lambda, \kappa)$-quasiisometrically in $H_{j}$ for some $j=j(i) \in\{1,2, \ldots, n\}$. The constants $(\lambda, \kappa)$ depend only on $(L, C)$ and on $(G, \mathcal{H})$.

Proof. If the group $G$ is finite then the group $G^{\prime}$ is also finite. We assume henceforth that both groups are infinite.

Let $\mathfrak{q}$ be an $(L, C)$-quasi-isometry from $G$ to $G^{\prime}$, and let $\bar{q}$ be its quasi-converse, such that $\operatorname{dist}\left(\mathfrak{q} \circ \overline{\mathfrak{q}}, \operatorname{id}_{G^{\prime}}\right) \leq D$ and $\operatorname{dist}\left(\overline{\mathfrak{q}} \circ \mathfrak{q}, \operatorname{id}_{G}\right) \leq D$, where $D=D(L, C)$. By Theorem 1.5, $G$ is ATG with respect to the collection of left cosets $\mathcal{A}=\left\{g H_{i} \mid\right.$ $\left.g \in G / H_{i}, i \in\{1,2, \ldots, n\}\right\}$. Theorem 5.1 in [DS05b] implies that $G^{\prime}$ is ATG with respect to $\mathfrak{q}(\mathcal{A})=\{\mathfrak{q}(A) \mid A \in \mathcal{A}\}$. Moreover all constants appearing in the properties $\left(\alpha_{i}\right), i=1,2,3$ (formulated in Theorem 4.9), $\left(\beta_{j}\right), j=2,3$, and (Qconv) (formulated in Theorem 4.22) for $\left(G^{\prime}, \mathfrak{q}(\mathcal{A})\right)$ can be expressed as functions of $(L, C)$ and of the constants in the similar properties for $(G, \mathcal{A})$.

Theorem 5.4 implies that $G^{\prime}$ is either hyperbolic or relatively hyperbolic with respect to a family of subgroups $\left\{H_{1}^{\prime}, \ldots, H_{m}^{\prime}\right\}$; moreover each $H_{i}^{\prime}$ is contained in $\mathcal{N}_{\mathcal{K}}\left(\mathfrak{q}\left(A_{i}\right)\right)$ for some $A_{i} \in \mathcal{A}$, where $\varkappa$ is a constant depending on $(L, C)$, on the constant $M$ in $\left(\beta_{2}\right)$ for $(G, \mathcal{A})$, and on the constant $\chi$ in $\left(\beta_{3}\right)$ for $(G, \mathcal{A})$ (see Remark 5.6). 
Let $\pi_{1}: \mathcal{N}_{\varkappa}\left(\mathfrak{q}\left(A_{i}\right)\right) \rightarrow \mathfrak{q}\left(A_{i}\right)$ be a map such that $\operatorname{dist}\left(x, \pi_{1}(x)\right) \leq \varkappa$. Then $\pi_{1}$ is a $(1,2 \varkappa)$-quasi-isometric embedding. Let $\pi_{2}: \mathcal{N}_{D}\left(A_{i}\right) \rightarrow A_{i}$ be a $(1,2 D)$-quasiisometric embedding constructed similarly. The restriction to $H_{i}^{\prime}$ of $\pi_{2} \circ \bar{q} \circ \pi_{1}$ is a $(\lambda, \kappa)$-quasi-isometric embedding of $H_{i}^{\prime}$ into $A_{i}=g H_{j}$, for some $j \in\{1,2, \ldots, n\}$, with $(\lambda, \kappa)$ depending on $(L, C), \varkappa$ and $D$.

If $G^{\prime}$ is hyperbolic then $G^{\prime}$ is relatively hyperbolic with respect to $\{1\} \neq\left\{G^{\prime}\right\}$ and all the statements in the theorem hold.

If $G^{\prime}=H_{i}^{\prime}$ then $G^{\prime}=\mathcal{N}_{\varkappa}\left(\mathfrak{q}\left(A_{i}\right)\right)$, which implies that $G \subset \mathcal{N}_{C}\left(\overline{\mathfrak{q}}\left(G^{\prime}\right)\right) \subset$ $\mathcal{N}_{L \varkappa+2 C+D}\left(A_{i}\right)$. By Theorem 1.5, this contradicts the fact that $G$ is (properly) hyperbolic relative to $\mathscr{H}$.

\section{References}

[AB05] E. Alibegovic and M. Bestvina, Limit groups are CAT(0). J. London Math. Soc. (2) 74 (2006), 259-272 Zbl 05057226 MR 2254564

[Beh05] J. Behrstock, Asymptotic geometry of the mapping class group and Teichmüller space. Geom. Topol. 10 (2006), 1523-1578 (electronic). Zbl 05117949 MR 2255505

[BDM05] J. Behrstock, C. Druţu, and L. Mosher, Thick metric spaces, relative hyperbolicity, and quasi-isometric rigidity. Math. Ann., to appear. arXiv:math.GT/0512592

[BF01] J. Brock and B. Farb, Curvature and rank of Teichmüller space. Amer. J. Math. 128 (2006), 1-22. Zbl 1092.32008 MR 2197066

[Bou65] N. Bourbaki, Éléments de mathématique. Fasc. II, Livre III: Topologie générale, Hermann, Paris 1965. Zbl 0102.37603 MR 0244924

[Bow97] B. Bowditch, Relatively hyperbolic groups. Preprint 1997, University of Southampton. http://www.maths.soton.ac.uk/pure/preprints.phtml

[Dah03a] F. Dahmani, Combination of convergence groups. Geom. Topology 7 (2003), 933-963. Zbl 1037.20042 MR 2026551

[Dah03b] F. Dahmani, Les groupes relativement hyperboliques et leurs bords. Ph.D. thesis, University of Strasbourg, 2003.

[dDW84] L. Van den Dries and A. Wilkie, On Gromov's theorem concerning groups of polynomial growth and elementary logic. J. Algebra 89 (1984), 349-374. Zbl 0552.20017 MR 0751150

[Dru02] C. Druţu, Quasi-isometry invariants and asymptotic cones. Int. J. Algebra Comput. 12 (2002), 99-135. Zbl 1010.20029 MR 1902363

[Dru04] C. Druţu, Quasi-isometry rigidity of groups. In Non-Positively Curved Geometries, Discrete Groups and Rigidity, Sémin. Congr. 18, Soc. Math. France, Paris 2008, 323-374.

[DS05a] C. Druţu and M. Sapir, Relatively hyperbolic groups with Rapid Decay property. Internat. Math. Res. Notices 19 (2005), 1181-1194. Zbl 1077.22006 MR 2147058 
Vol. 84 (2009) Relatively hyperbolic groups: geometry and quasi-isometric invariance

[DS05b] C. Druţu and M. Sapir, Tree-graded spaces and asymptotic cones of groups. With an appendix by D. Osin and M. Sapir. Topology 44 (2005), 959-1058. Zbl 1101.20025 MR 2153979

[Dyu00] A. Dyubina, Instability of the virtual solvability and the property of being virtually torsion-free for quasi-isometric groups. Internat. Math. Res. Notices 21 (2000), 1097-1101. Zbl 0979.20034 MR 1800990

[EFW05] A. Eskin, D. Fisher and K. Whyte, Quasi-isometries and rigidity of solvable groups. Pure Appl. Math. Q. 3 (2007), 927-947. Zbl 05296093 MR 2402598

[Far97] B. Farb, The quasi-isometry classification of lattices in semisimple Lie groups. Math. Res. Lett. 4 (1997), 705-717. Zbl 0889.22010 MR 1484701

[Far98] B. Farb, Relatively hyperbolic groups. Geom. Funct. Anal. 8 (1998), 810-840. Zbl 0985.20027 MR 1650094

[FM98] B. Farb and L. Mosher, .A rigidity theorem for the solvable Baumslag-Solitar groups. Appendix by D. Cooper. Invent. Math. 131 (1998), 419-451,Zbl 0937.22003 MR 1608595

[FM99] B. Farb and L. Mosher, Quasi-isometric rigidity for the solvable Baumslag-Solitar groups, II. Invent. Math. 137 (1999), 613-649. Zbl 0931.20035 MR 1709862

[GdlH90] E. Ghys and P. de la Harpe (eds.), Sur les groupes hyperboliques d'après Mikhael Gromov. Progr. Math. 83, Birkhäuser, Boston 1990. Zbl 0731.20025 MR 1086648

[Gro81] M. Gromov, Groups of polynomial growth and expanding maps. Inst. Hautes Études Sci. Publ. Math. 53 (1981), 53-73. Zbl 0474.20018 MR 0623534

[Gro87] M. Gromov, Hyperbolic groups, Essays in group theory (S. Gersten, ed.), MSRI Publications 8, Springer-Verlag, 1987. Zbl 0634.20015 MR 0919829

[Gro93] M. Gromov, Asymptotic invariants of infinite groups. In Geometric Group Theory (Sussex, 1991), Vol. 2, G. Niblo and M. Roller, eds., London Math. Soc. Lecture Note Ser. 182, Cambridge University Press, Cambridge 1993, 1-295. Zbl 0841.20039 MR 1253544

[Hru04] C. Ch. Hruska, Nonpositively curved 2-complexes with isolated flats. Geom. Topol. 8 (2004), 205-275. Zbl 1063.20048 MR 2033482

[HK05] C. Ch. Hruska and B. Kleiner, Hadamard spaces with isolated flats. Geom. Topol. 9 (2005), 1501-1538. Zbl 1087.20034 MR 2175151

[KL95] M. Kapovich and B. Leeb, On asymptotic cones and quasi-isometry classes of fundamental groups of nonpositively curved manifolds. Geom. Funct. Anal. 5 (1995), 582-603. Zbl 0829.57006 MR 1339818

[KL97] M. Kapovich and B. Leeb, Quasi-isometries preserve the geometric decomposition of Haken manifolds. Invent. Math. 128 (1997), 393-416. Zbl 0866.20033 MR 1440310

[KL98] M. Kapovich and B. Leeb, 3-manifold groups and nonpositive curvature. Geom. Funct. Anal. 8 (1998), 841-852. Zbl 0915.57009 MR 1650098

[KKL98] M. Kapovich, B. Kleiner and B. Leeb, Quasi-isometries and the de Rham decomposition. Topology 37 (1998), 1193-1211. Zbl 0954.53027 MR 1632904

[KS04] I. Kapovich and P. Schupp, Relative hyperbolicity and Artin groups. Geom. Dedicata 107 (2004), 153-167. Zbl 1080.20032 MR 2110760 
[OOS06] A. Yu. Ol'shanskii, D. V. Osin and M. V. Sapir, Lacunary hyperbolic groups, Preprint, 2007. arXiv:math.GR/0701365

[Osi04] D. V. Osin, Small cancellations over relatively hyperbolic groups and embedding theorems, Preprint, 2004. arXiv:math.GR/0411039

[Osi06] D. V. Osin, Relatively hyperbolic groups: intrinsic geometry, algebraic properties, and algorithmic problems. Mem. Amer. Math. Soc. 179 (2006), no. 843. Zbl 1093.20025 MR 2182268

[PW02] P. Papasoglu and K. Whyte, Quasi-isometries between groups with infinitely many ends. Comment. Math. Helv. 77 (2002), 133-144. Zbl 1010.20026 MR 1898396

[Sch96] R. Schwartz, The quasi-isometry classification of rank one lattices. Inst. Hautes Études Sci. Publ. Math. 82 (1996), 133-168. Zbl 0852.22010 MR 1383215

[Yam04] A. Yaman, A topological characterisation of relatively hyperbolic groups. J. Reine Angew. Math. 566 (2004), 41-89. Zbl 1043.20020 MR 2039323

Received July 12, 2007

Cornelia Druţu, Mathematical Institute, 24-29 St Giles, Oxford OX1 3LB, United Kingdom E-mail: drutu@maths.ox.ac.uk 